\title{
Prevalence of small vessel and large vessel disease in diabetic patients from 14 centres
}

\section{The World Health Organisation Multinational Study of Vascular Disease in Diabetics}

\author{
Prepared by: Diabetes Drafting Group
}

\section{Contents}

\section{Summary}

Introduction

Study populations

1. Recruitment of centres and samples

2. Sampling procedures

Materials and methods

1. Data collection and laboratory procedures

2. Large-vessel disease endpoints

3. Small-vessel disease endpoints

4. Statistical procedures

Results

1. Age, sex and duration composition

2. Biometric, clinical and biochemical characteristics

3. Large-vessel disease

(a) Prevalence overall and by centre

(b) Univariate and multivariate associations
4. Small-vessel disease

(a) Prevalence overall and by centre

(b) Univariate and multivariate associations

Discussion

Recruitment

Vascular 'risk factors'

Treatment of diabetes mellitus

Large-vessel disease prevalence

Small-vessel disease prevalence

Implications of study

The interpretations and comments are those of the authors alone, and this report is not an official publication of the World Health Organisation.
Summary. A total of 6695 diabetic men and women, aged 35 to 54 years, from 14 centres and representing 13 national groups, participated in a vascular disease prevalence survey. A random sample was drawn after stratification of each centre's diabetic base population by sex, duration of diabetes and age. A common agreed protocol, standardized examination procedures, and centralized laboratory methods were used in the investigation. Within the age range examined there was considerable variation between centres in a number of variables, including degree of obesity (measured as Body Mass Index (BMI)), proportion treated with insulin and proportion of cigarette smokers. The latter also showed considerable sex differences within centres. Subjects with age at onset below 25 years were notably few in Hong Kong, Tokyo and Oklahoma. There was also considerable variation in the apparent prevalence of both large- and small-vessel (macrovascular and microvascular) disease between centres. In pooled data, measures of large-vessel disease were significantly and independently associated with age, blood pressure and BMI in both sexes, and with diabetes duration and plasma cholesterol in men only. Within-centre analyses showed blood pressure to be the most consistently associated variable in both sexes. In pooled data, small-vessel disease of the eye was significantly and independently associated with diabetes duration, blood pressure, BMI and type of treatment in both sexes. In withincentre analyses, diabetes duration was the most consistently associated variable, followed by blood pressure. Proteinuria as an index of small-vessel disease of the kidney was, in pooled data, significantly and independently correlated with diabetes duration, blood pressure and plasma cholesterol in both sexes. In within-centre analyses, blood pressure was the most consistently associated variable, with diabetes duration and plasma cholesterol equal second - significant in 12 of the 28 centre/sex groups. Heterogeneity of large-vessel disease prevalence in diabetic subjects is confirmed by this study, and the possibility of heterogeneity in small-vessel disease prevalence and severity is suggested.

Key words: Diabetes mellitus, atherosclerosis, retinopathy, nephropathy, complications, epidemiology. 
Table 1. Participation goals: numbers of diabetic subjects of each sex, subdivided by duration and age-group, to be recruited by each centre

\begin{tabular}{llllc}
\hline $\begin{array}{l}\text { Diabetes dura- } \\
\text { tion (years) }\end{array}$ & $35-41$ & Age (years) & \multicolumn{2}{c}{ Total } \\
\cline { 2 - 3 } & & $42-48$ & $49-54$ & \\
\hline $1-6$ & 28 & 28 & 28 & 84 \\
$7-13$ & 28 & 28 & 28 & 84 \\
$14+$ & 28 & 28 & 28 & 84 \\
\hline All & 84 & 84 & 84 & 252 \\
\hline
\end{tabular}

Table 2. Definitions of large-vessel disease (LVD): components, composites, and subclasses

\begin{tabular}{|c|c|c|c|c|c|}
\hline \multirow{2}{*}{$\begin{array}{l}\text { Components } \\
\text { of large-vessel } \\
\text { disease }\end{array}$} & \multicolumn{3}{|c|}{$\begin{array}{l}\text { Composites of } \\
\text { large-vessel disease }\end{array}$} & \multicolumn{2}{|c|}{$\begin{array}{l}\text { Subclasses of } \\
\text { large-vessel disease }\end{array}$} \\
\hline & HVD & Leg VD & LVD & $\begin{array}{l}\text { CVD } \\
\text { Severe }\end{array}$ & $\begin{array}{l}\text { CVD } \\
\text { Intermediate }\end{array}$ \\
\hline Angina pectoris & $* *$ & & $* *$ & & $* *$ \\
\hline Possible infarction & $* *$ & & $* *$ & & $* *$ \\
\hline $\begin{array}{l}\text { Intermittent } \\
\text { claudication }\end{array}$ & & $* *$ & $* *$ & & $* *$ \\
\hline $\begin{array}{l}\text { Amputation } \\
\text { or gangrene }\end{array}$ & & $* *$ & ** & $* *$ & $* *$ \\
\hline Stroke & & & $* *$ & & $* *$ \\
\hline $\begin{array}{l}\text { ECG coronary } \\
\text { probable }\end{array}$ & $* *$ & & $* *$ & $* *$ & \\
\hline $\begin{array}{l}\text { ECG coronary } \\
\text { possible }\end{array}$ & $* *$ & & $* *$ & & $* *$ \\
\hline
\end{tabular}

Asterisks under each sub-head indicate the components which are included. HVD - heart vascular disease; Leg VD - leg vascular disease, LVD - large vessel disease; CVD - cardiovascular disease

Table 3. Definitions of small vessel disease (SVD): components, composites, and subclasses

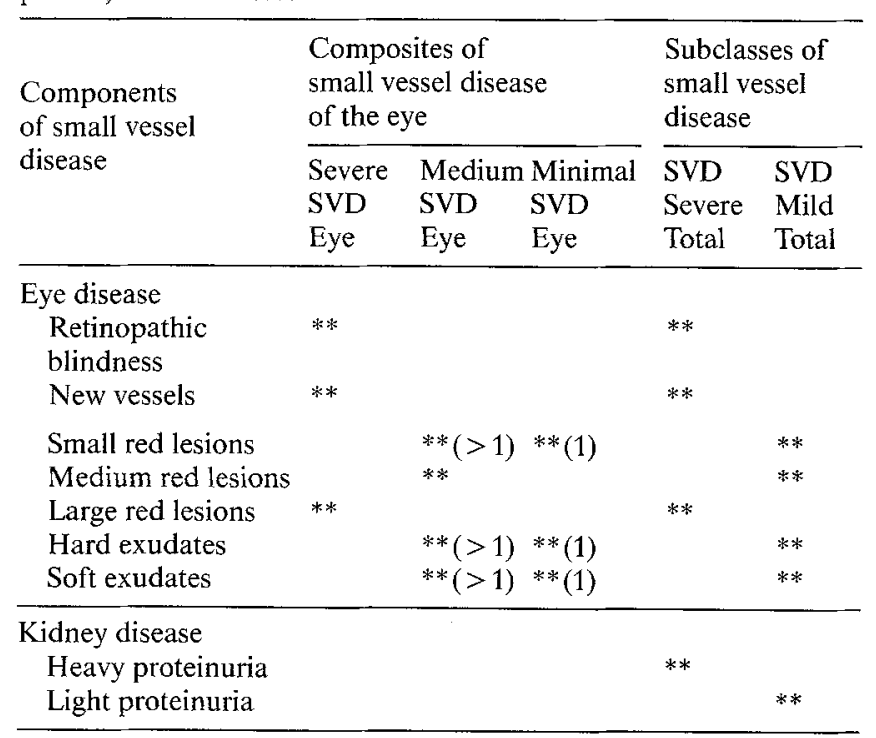

Asterisks under each sub-head indicate the components which are included. NB: Both eye and kidney disease components are required for inclusion in the 'severe' and 'mild' SVD Subclasses

Vascular disease of the retina and kidney typifies the long-term diabetic state, and is generally assumed to vary little in prevalence among diverse groups of patients with diabetes of comparable duration. By contrast,
Table 4. Numbers recruited at each centre, by sex

\begin{tabular}{llccc}
\hline Centre & Centre code & Male & Female & Total \\
\hline London & (LON) & 254 & 243 & 497 \\
Switzerland & (SWI) & 278 & 256 & 534 \\
Brussels & (BRU) & 236 & 186 & 422 \\
Moscow & (MOS) & 226 & 273 & 499 \\
Warsaw & (WAR) & 241 & 245 & 486 \\
Berlin & (BER) & 285 & 275 & 560 \\
Zagreb & (ZAG) & 222 & 180 & 402 \\
New Delhi & (DEL) & 289 & 266 & 555 \\
Hong Kong & (HON) & 198 & 224 & 422 \\
Tokyo & (TOK) & 235 & 201 & 436 \\
Havana & (HAV) & 258 & 257 & 515 \\
Oklahoma & (OKL) & 270 & 383 & 653 \\
Arizona & (ARI) & 87 & 154 & 241 \\
Bulgaria & (BUL) & 222 & 251 & 473 \\
\hline Total & & 3301 & 3394 & 6695 \\
\hline
\end{tabular}

Table 5. Proportions (\%) of centre totals by sex and age at interview

\begin{tabular}{|c|c|c|c|c|}
\hline \multirow[t]{2}{*}{ Centre } & \multirow[t]{2}{*}{ Sex } & \multicolumn{3}{|c|}{ Age at interview } \\
\hline & & $35-41$ years & $42-48$ years & $49-54$ years \\
\hline \multirow[t]{2}{*}{ LON } & Male & 28.0 & 29.9 & 42.1 \\
\hline & Female & 21.8 & 35.8 & 42.4 \\
\hline \multirow[t]{2}{*}{ SWI } & Male & 32.4 & 35.3 & 32.4 \\
\hline & Female & 34.0 & 34.8 & 31.3 \\
\hline \multirow[t]{2}{*}{ BRU } & Male & 25.4 & 30.1 & 44.5 \\
\hline & Female & 31.2 & 30.1 & 38.7 \\
\hline \multirow[t]{2}{*}{ MOS } & Male & 23.0 & 34.5 & 42.5 \\
\hline & Female & 27.5 & 39.6 & 33.0 \\
\hline \multirow[t]{2}{*}{ WAR } & Male & 21.6 & 33.2 & 45.2 \\
\hline & Female & 23.7 & 34.3 & 42.0 \\
\hline \multirow[t]{2}{*}{ BER } & Male & 29.1 & 31.6 & 39.3 \\
\hline & Female & 26.9 & 32.7 & 40.4 \\
\hline \multirow[t]{2}{*}{$\mathrm{ZAG}$} & Male & 29.3 & 32.4 & 38.3 \\
\hline & Female & 21.1 & 38.9 & 40.0 \\
\hline \multirow[t]{2}{*}{ DEL } & Male & 30.8 & 35.3 & 33.9 \\
\hline & Female & 25.6 & 38.0 & 36.5 \\
\hline \multirow[t]{2}{*}{$\mathrm{HON}$} & Male & 14.1 & 34.8 & 51.0 \\
\hline & Female & 26.3 & 26.8 & 46.9 \\
\hline \multirow[t]{2}{*}{ TOK } & Male & 21.3 & 34.9 & 43.8 \\
\hline & Female & 19.4 & 28.4 & 52.2 \\
\hline \multirow[t]{2}{*}{ HAV } & Male & 30.6 & 35.7 & 33.7 \\
\hline & Female & 33.5 & 34.2 & 32.3 \\
\hline \multirow[t]{2}{*}{ OKL } & Male & 24.1 & 34.8 & 41.1 \\
\hline & Female & 22.5 & 37.6 & 39.9 \\
\hline \multirow[t]{2}{*}{ ARI } & Male & 27.6 & 33.3 & 39.1 \\
\hline & Female & 20.1 & 35.7 & 44.2 \\
\hline \multirow[t]{2}{*}{ BUL } & Male & 23.0 & 29.3 & 47.7 \\
\hline & Female & 25.1 & 31.9 & 43.0 \\
\hline \multirow[t]{2}{*}{ Total } & Male & 26.0 & 33.3 & 40.7 \\
\hline & Female & 25.8 & 34.4 & 39.8 \\
\hline
\end{tabular}

ischaemic disease of heart, leg and brain tissues (often subsumed under the unsatisfactory terms 'atherosclerotic' or 'macrovascular' disease), though generally accepted as more commonly affecting the diabetic than 
Table 6. Proportions (\%) of centre totals by sex and diabetes duration at interview

\begin{tabular}{|c|c|c|c|c|}
\hline \multirow[t]{2}{*}{ Centre } & \multirow[t]{2}{*}{$\operatorname{Sex}$} & \multicolumn{3}{|c|}{ Diabetes duration } \\
\hline & & $0-6$ years & $7-13$ years & $14+$ years \\
\hline LON & $\begin{array}{l}\text { Male } \\
\text { Female }\end{array}$ & $\begin{array}{l}29.5 \\
28.8\end{array}$ & $\begin{array}{l}32.3 \\
32.9\end{array}$ & $\begin{array}{l}38.2 \\
38.3\end{array}$ \\
\hline SWI & $\begin{array}{l}\text { Male } \\
\text { Female }\end{array}$ & $\begin{array}{l}34.5 \\
34.0\end{array}$ & $\begin{array}{l}32.0 \\
30.1\end{array}$ & $\begin{array}{l}33.5 \\
35.9\end{array}$ \\
\hline BRU & $\begin{array}{l}\text { Male } \\
\text { Female }\end{array}$ & $\begin{array}{l}49.2 \\
46.2\end{array}$ & $\begin{array}{l}22.0 \\
22.0\end{array}$ & $\begin{array}{l}28.8 \\
31.7\end{array}$ \\
\hline MOS & $\begin{array}{l}\text { Male } \\
\text { Fermale }\end{array}$ & $\begin{array}{l}33.6 \\
36.6\end{array}$ & $\begin{array}{l}40.7 \\
42.1\end{array}$ & $\begin{array}{l}25.7 \\
21.2\end{array}$ \\
\hline WAR & $\begin{array}{l}\text { Male } \\
\text { Female }\end{array}$ & $\begin{array}{l}31.5 \\
29.8\end{array}$ & $\begin{array}{l}31.5 \\
29.0\end{array}$ & $\begin{array}{l}36.9 \\
41.2\end{array}$ \\
\hline BER & $\begin{array}{l}\text { Male } \\
\text { Female }\end{array}$ & $\begin{array}{l}30.9 \\
32.4\end{array}$ & $\begin{array}{l}33.7 \\
34.5\end{array}$ & $\begin{array}{l}35.4 \\
33.1\end{array}$ \\
\hline $\mathrm{ZAG}$ & $\begin{array}{l}\text { Male } \\
\text { Female }\end{array}$ & $\begin{array}{l}44.1 \\
48.9\end{array}$ & $\begin{array}{l}30.6 \\
33.9\end{array}$ & $\begin{array}{l}25.2 \\
17.2\end{array}$ \\
\hline DEL & $\begin{array}{l}\text { Male } \\
\text { Female }\end{array}$ & $\begin{array}{l}38.4 \\
45.1\end{array}$ & $\begin{array}{l}36.0 \\
32.7\end{array}$ & $\begin{array}{l}25.6 \\
22.2\end{array}$ \\
\hline HON & $\begin{array}{l}\text { Male } \\
\text { Female }\end{array}$ & $\begin{array}{l}49.5 \\
46.0\end{array}$ & $\begin{array}{l}28.8 \\
37.1\end{array}$ & $\begin{array}{l}21.7 \\
17.0\end{array}$ \\
\hline TOK & $\begin{array}{l}\text { Male } \\
\text { Female }\end{array}$ & $\begin{array}{l}38.7 \\
43.8\end{array}$ & $\begin{array}{l}38.3 \\
36.8\end{array}$ & $\begin{array}{l}23.0 \\
19.4\end{array}$ \\
\hline HAV & $\begin{array}{l}\text { Male } \\
\text { Female }\end{array}$ & $\begin{array}{l}34.1 \\
33.5\end{array}$ & $\begin{array}{l}36.0 \\
35.4\end{array}$ & $\begin{array}{l}29.8 \\
31.1\end{array}$ \\
\hline OKL & $\begin{array}{l}\text { Male } \\
\text { Female }\end{array}$ & $\begin{array}{l}64.8 \\
65.3\end{array}$ & $\begin{array}{l}25.2 \\
21.9\end{array}$ & $\begin{array}{l}10.0 \\
12.8\end{array}$ \\
\hline ARI & $\begin{array}{l}\text { Male } \\
\text { Female }\end{array}$ & $\begin{array}{l}36.8 \\
31.2\end{array}$ & $\begin{array}{l}47.1 \\
46.1\end{array}$ & $\begin{array}{l}16.1 \\
22.7\end{array}$ \\
\hline BUL & $\begin{array}{l}\text { Male } \\
\text { Female }\end{array}$ & $\begin{array}{l}42.8 \\
38.2\end{array}$ & $\begin{array}{l}31.1 \\
34.7\end{array}$ & $\begin{array}{l}26.1 \\
27.1\end{array}$ \\
\hline Total & $\begin{array}{l}\text { Male } \\
\text { Female }\end{array}$ & $\begin{array}{l}39.8 \\
40.8\end{array}$ & $\begin{array}{l}32.6 \\
32.9\end{array}$ & $\begin{array}{l}27.5 \\
26.3\end{array}$ \\
\hline
\end{tabular}

the non-diabetic person, is thought to vary widely in prevalence among geographically distinct groups of diabetic subjects, especially when oriental and occidental or North and South comparisons are made. For example, several autopsy and clinical reports from Japan since the late 1960s suggest that coronary heart disease occurs at rates substantially lower than those familiar to European and American diabetes centres [1]. The diabetic populations appear to mirror, though at an aggravated level of intensity, the variations in arterial disease prevalence between the corresponding non-diabetic populations $[1,2]$.

The need to confirm, quantify and explain these reports of widely differing atherosclerosis rates, and to make valid comparisons of prevalence of microangiopathy, prompted a proposal in the early 1970 s to compare vascular disease in diabetes in a Tokyo-London standardized study. This project attracted the interest of others and was widened to allow multinational participation. Representatives from a number of national groups, the European Diabetes Epidemiology Study Group, the
Table 7. Distribution (\%) of age at diagnosis of diabetes by sex and centre

\begin{tabular}{|c|c|c|c|c|c|c|c|c|}
\hline \multirow[t]{2}{*}{ Centre } & \multicolumn{4}{|c|}{ Male (years) } & \multicolumn{4}{|c|}{ Female (years) } \\
\hline & $<25$ & $25-34$ & $35-44$ & $45-54$ & $<25$ & $25-34$ & $35-44$ & $45-54$ \\
\hline LON & 22.0 & 25.2 & 37.4 & 15.4 & 24.7 & 25.5 & 32.9 & 16.9 \\
\hline SWI & 17.3 & 31.3 & 36.3 & 15.1 & 20.7 & 25.4 & 40.6 & 13.3 \\
\hline BRU & 13.1 & 21.2 & 36.0 & 29.7 & 21.0 & 22.6 & 25.8 & 30.6 \\
\hline MOS & 11.1 & 24.3 & 43.4 & 21.2 & 6.6 & 30.8 & 44.0 & 18.7 \\
\hline WAR & 14.5 & 27.8 & 36.5 & 21.2 & 13.1 & 32.7 & 36.7 & 17.6 \\
\hline BER & 11.9 & 31.9 & 42.5 & 13.7 & 15.6 & 25.8 & 43.3 & 15.3 \\
\hline ZAG & 6.8 & 27.9 & 40.1 & 25.2 & 3.8 & 20.0 & 45.0 & 31.1 \\
\hline DEL & 8.3 & 34.3 & 42.6 & 14.9 & 3.8 & 30.8 & 44.4 & 21.1 \\
\hline HON & 1.5 & 15.2 & 54.5 & 28.8 & 4.9 & 16.1 & 53.1 & 25.9 \\
\hline TOK & 2.1 & 29.8 & 49.8 & 18.3 & 6.5 & 19.9 & 46.3 & 27.4 \\
\hline $\mathrm{HAV}$ & 11.2 & 36.0 & 39.1 & 13.6 & 14.8 & 32.7 & 39.3 & 13.2 \\
\hline $\mathrm{OKL}$ & 2.2 & 21.5 & 42.6 & 33.7 & 1.8 & 22.7 & 47.5 & 27.9 \\
\hline ARI & 5.7 & 32.2 & 47.1 & 14.9 & 3.2 & 32.5 & 50.0 & 14.3 \\
\hline BUL & 7.2 & 23.0 & 46.8 & 23.0 & 8.8 & 29.5 & 43.0 & 18.7 \\
\hline Total & 10.1 & 27.4 & 42.0 & 20.5 & 10.5 & 26.3 & 42.4 & 20.7 \\
\hline
\end{tabular}

International Diabetes Federation and the World Health Organisation (WHO) met in the mid-1970s to develop a multinational collaborative study. The final plan was formulated under the auspices of the WHO, which provided facilities and funds for central coordination, data collection and analysis, working meetings of participants and continuing skilled administrative help. Fourteen centres from thirteen countries ultimately participated in the study, each raising local support for the field work.

This report presents the prevalence data on largeand small-vessel disease, collected and analysed at the completion date for this WHO Multinational Study of Vascular Disease in Diabetics. The study serves the dual purpose of comparing morbidity in the participating diabetic groups and providing baseline data for future comparative estimates of incident events among them.

\section{Study populations}

\section{Recruitment of centres and samples}

The 14 centres participating in the study represented a diverse geographical, ethnic and cultural mix. They comprised three from Asia, a cluster from Europe and two indigenous Amerindian populations from North America (Table 1). Regrettably, no African group could be recruited.

Study patients were recruited from different sources. Each centre was asked to select the source that would best meet the requirements for representativity. In several centres samples were recruited from outpatient clinics serving populations typical of the area, as in the case of London, Brussels, Moscow, Warsaw, New Delhi, Hong Kong, Tokyo and Bulgaria. In Moscow the sample was randomly drawn from a number of selected clinics within a centrally coordinated health system. The Berlin (GDR), Zagreb, Havana and Oklahoma patients were sampled from a geographically defined register of all diabetics residing within a circumscribed area or belonging to an 
Table 8. Proportional distribution (\%) of systolic blood pressure $(\mathrm{mmHg})$ by sex and centre

\begin{tabular}{|c|c|c|c|c|c|c|c|c|c|c|}
\hline \multirow[b]{2}{*}{ Centre } & \multicolumn{5}{|l|}{ Male } & \multicolumn{5}{|c|}{ Female } \\
\hline & $<120$ & $120-139$ & $140-159$ & $160-179$ & $180+$ & $<120$ & $120-139$ & $140-159$ & $160-179$ & $180+$ \\
\hline LON & 21.3 & 44.5 & 21.7 & 9.4 & 3.1 & 21.8 & 36.2 & 22.2 & 11.1 & 8.6 \\
\hline SWI & 13.3 & 42.4 & 30.2 & 9.0 & 5.0 & 13.7 & 31.6 & 36.7 & 12.5 & 5.5 \\
\hline BRU & 22.5 & 36.9 & 24.6 & 10.6 & 5.5 & 15.6 & 26.3 & 30.1 & 18.8 & 8.6 \\
\hline MOS & 10.2 & 42.9 & 28.8 & 11.5 & 6.6 & 14.3 & 32.6 & 27.1 & 13.2 & 12.8 \\
\hline WAR & 18.7 & 32.8 & 29.5 & 12.9 & 6.2 & 16.7 & 26.5 & 32.7 & 14.3 & 9.8 \\
\hline BER & 13.3 & 34.4 & 27.4 & 14.4 & 10.5 & 14.5 & 32.7 & 29.1 & 16.7 & 6.9 \\
\hline $\mathrm{ZAG}$ & 8.6 & 33.8 & 32.9 & 16.7 & 7.2 & 1.7 & 22.2 & 41.1 & 21.1 & 11.7 \\
\hline DEL & 23.9 & 38.1 & 24.2 & 9.7 & 4.2 & 14.7 & 35.0 & 32.3 & 12.4 & 5.6 \\
\hline $\mathrm{HON}$ & 12.6 & 33.3 & 31.3 & 14.6 & 8.1 & 12.1 & 39.3 & 21.9 & 16.1 & 10.7 \\
\hline TOK & 30.2 & 37.0 & 23.8 & 7.7 & 1.3 & 26.4 & 32.8 & 28.4 & 9.5 & 3.0 \\
\hline $\mathrm{HAV}$ & 17.1 & 36.0 & 26.0 & 14.0 & 7.0 & 14.8 & 28.0 & 29.2 & 18.7 & 9.3 \\
\hline OKL & 15.2 & 45.6 & 28.9 & 7.8 & 2.6 & 21.1 & 42.3 & 23.5 & 10.4 & 2.6 \\
\hline ARI & 35.6 & 41.4 & 17.2 & 3.4 & 2.3 & 48.1 & 29.9 & 16.2 & 3.9 & 1.3 \\
\hline BUL & 19.4 & 37.8 & 25.2 & 10.8 & 6.8 & 15.1 & 37.8 & 26.3 & 15.1 & 5.6 \\
\hline Total & 18.0 & 38.4 & 26.9 & 11.1 & 5.6 & 17.4 & 33.1 & 28.3 & 13.8 & 7.2 \\
\hline
\end{tabular}

Table 9. Hypertension prevalence by sex and centre

\begin{tabular}{|c|c|c|c|c|}
\hline Centre & Sex & $\begin{array}{l}\text { Subjects hy- } \\
\text { pertensive } \\
\text { (who defini- } \\
\text { tion) }(\%)\end{array}$ & $\begin{array}{l}\text { Subjects with } \\
\text { normal systolic } \\
\text { and diastolic BP } \\
\text { but on BP-low- } \\
\text { ering treatment } \\
(\%)\end{array}$ & $\begin{array}{l}\text { Subjects on BP- } \\
\text { lowering treat- } \\
\text { ment }(\%)\end{array}$ \\
\hline LON & $\begin{array}{l}\text { Male } \\
\text { Female }\end{array}$ & $\begin{array}{l}22.0 \\
29.6\end{array}$ & $\begin{array}{l}2.4 \\
3.3\end{array}$ & $\begin{array}{l}4.3 \\
7.8\end{array}$ \\
\hline SWI & $\begin{array}{l}\text { Male } \\
\text { Female }\end{array}$ & $\begin{array}{l}33.8 \\
38.7\end{array}$ & $\begin{array}{r}6.5 \\
10.2\end{array}$ & $\begin{array}{l}15.8 \\
22.3\end{array}$ \\
\hline BRU & $\begin{array}{l}\text { Male } \\
\text { Female }\end{array}$ & $\begin{array}{l}30.9 \\
38.7\end{array}$ & $\begin{array}{l}1.3 \\
0.5\end{array}$ & $\begin{array}{l}4.7 \\
3.2\end{array}$ \\
\hline MOS & $\begin{array}{l}\text { Male } \\
\text { Female }\end{array}$ & $\begin{array}{l}39.4 \\
46.9\end{array}$ & $\begin{array}{l}18.1 \\
17.9\end{array}$ & $\begin{array}{l}31.9 \\
39.2\end{array}$ \\
\hline WAR & $\begin{array}{l}\text { Male } \\
\text { Female }\end{array}$ & $\begin{array}{l}29.0 \\
35.5\end{array}$ & $\begin{array}{l}2.5 \\
5.3\end{array}$ & $\begin{array}{l}12.4 \\
19.2\end{array}$ \\
\hline BER & $\begin{array}{l}\text { Male } \\
\text { Female }\end{array}$ & $\begin{array}{l}38.6 \\
36.0\end{array}$ & $\begin{array}{l}3.2 \\
7.3\end{array}$ & $\begin{array}{l}15.4 \\
21.5\end{array}$ \\
\hline ZAG & $\begin{array}{l}\text { Male } \\
\text { Female }\end{array}$ & $\begin{array}{l}36.0 \\
48.9\end{array}$ & $\begin{array}{r}5.9 \\
11.7\end{array}$ & $\begin{array}{l}13.1 \\
30.6\end{array}$ \\
\hline DEL & $\begin{array}{l}\text { Male } \\
\text { Female }\end{array}$ & $\begin{array}{l}26.6 \\
33.1\end{array}$ & $\begin{array}{l}6.2 \\
7.9\end{array}$ & $\begin{array}{l}15.2 \\
18.0\end{array}$ \\
\hline $\mathrm{HON}$ & $\begin{array}{l}\text { Male } \\
\text { Female }\end{array}$ & $\begin{array}{l}27.8 \\
30.8\end{array}$ & $\begin{array}{l}2.5 \\
2.7\end{array}$ & $\begin{array}{l}12.6 \\
13.4\end{array}$ \\
\hline TOK & $\begin{array}{l}\text { Male } \\
\text { Female }\end{array}$ & $\begin{array}{l}28.9 \\
27.4\end{array}$ & $\begin{array}{r}7.2 \\
11.4\end{array}$ & $\begin{array}{l}16.6 \\
19.4\end{array}$ \\
\hline HAV & $\begin{array}{l}\text { Male } \\
\text { Female }\end{array}$ & $\begin{array}{l}31.0 \\
40.9\end{array}$ & $\begin{array}{l}3.5 \\
5.1\end{array}$ & $\begin{array}{l}10.1 \\
21.4\end{array}$ \\
\hline OKL & $\begin{array}{l}\text { Male } \\
\text { Female }\end{array}$ & $\begin{array}{l}35.9 \\
34.7\end{array}$ & $\begin{array}{l}18.1 \\
17.2\end{array}$ & $\begin{array}{l}24.8 \\
23.5\end{array}$ \\
\hline ARI & $\begin{array}{l}\text { Male } \\
\text { Female }\end{array}$ & $\begin{array}{l}33.3 \\
26.0\end{array}$ & $\begin{array}{l}20.7 \\
18.8\end{array}$ & $\begin{array}{l}27.6 \\
22.7\end{array}$ \\
\hline BUL & $\begin{array}{l}\text { Male } \\
\text { Female }\end{array}$ & $\begin{array}{l}32.0 \\
35.1\end{array}$ & $\begin{array}{l}6.3 \\
9.6\end{array}$ & $\begin{array}{l}18.9 \\
23.1\end{array}$ \\
\hline Total & $\begin{array}{l}\text { Male } \\
\text { Female } \\
\text { Total }\end{array}$ & $\begin{array}{l}31.8 \\
36.0 \\
33.9\end{array}$ & $\begin{array}{l}6.8 \\
9.4 \\
8.2\end{array}$ & $\begin{array}{l}15.4 \\
20.8 \\
18.1\end{array}$ \\
\hline
\end{tabular}

identifiable community group (e.g. Pima Indians). The Swiss sample collected through a large number of local practitioners was representative of an even larger area including almost the entire country. For want of numbers a few centres extended their recruitment to further defined patient populations after seeking agreement from the WHO coordinating team. Otherwise centres did not deviate from the recruitment plan defined earlier $[3,4]$. The list of centres and investigators appears in Appendix 1.

The study protocol required equal numbers of men and women with diabetes, sampled from three age bands within the range 35 to 54 years. This range was chosen for two main reasons. Arterial disease, in particular coronary heart disease, is very uncommon below age 35 ; after 54 years selective mortality could distort its representation in different diabetic populations. The known duration of diabetes is another major variable, clearly associated with microvascular disease, so sampling was also structured to ensure a range of duration within the selected sex and age groups.

For this investigation a diabetic was defined as a patient diagnosed at least 1 year earlier and under treatment for the disorder by the physicians of the participating centre at a defined date. This working definition circumvented the difficult task of obtaining diagnostic criteria agreed by all participants. Its use may have resulted in the inclusion of a very few non-diabetic subjects, but all of the clinics were served by highly experienced clinicians unlikely to have made many gross diagnostic errors. The specific exclusion of recruitment by population screening was intended to minimize the inclusion of early or questionably diabetic patients. Also excluded were 'specialist' clinics known to attract patients referred, for example, because of particular complications of diabetes.

Potential sources of bias were also monitored by recording the manner of diagnostic presentation and the path of referral. Specific questions were designed to indicate clinical 'severity' (insulin-dependence).

\section{Sampling procedures}

Casual acquisition of subjects gives selective advantage to frequent or complicated clinic attenders. This bias was minimized by sampling from a register constructed of all patients, in the appropriate age categories, who had attended once or more in the year before the commencement of the study.

The sample size was a compromise between the constraints of the collection exercise itself (practicability and resources available), the number of patients likely to be available to fill the most difficult 'cells' of the recruiting matrix, and the need to ensure adequate statistical power to demonstrate differences of given dimensions between the frequencies of the key endpoints in the various centres. 
Table 10. Mean systolic blood pressure $(\mathrm{mmHg})$ by age group sex and centre

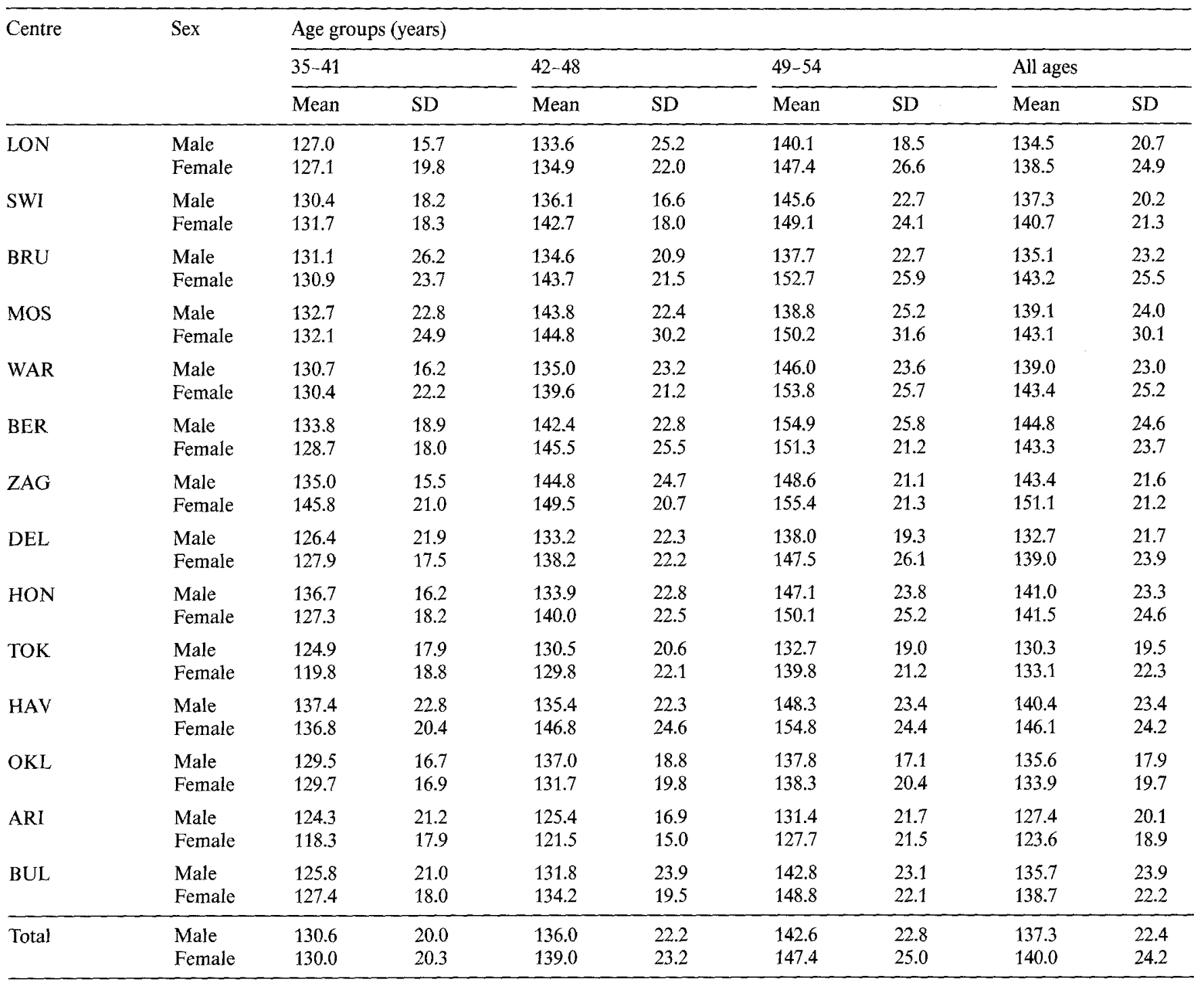

The agreed target number for each centre was 252 men and 252 women, distributed by age and diabetes duration as shown in Table 1. Twenty-eight patients were randomly selected from the recruitment register for each cell; a further seven were reserved to draw from if necessary. Some centres had comparatively few youthful, long-duration patients, making certain cells difficult to fill and for such cases the source of recruitment was extended with WHO agreement. Identifiable minorities differing from the prevailing ethnic or cultural patterns were excluded. Two centres departed from these sampling procedures. The Oklahoma sample had been suitably drawn for other purposes before the WHO study began, and the Arizona sample included all available subjects.

Consent was obtained before enrolment from each individual selected for study. Participating groups were permitted to add "noncore' investigations, either on a unilateral basis or as part of joint enterprises, so long as they did not prejudice the major purposes of the study. These included local investigations in which vascular disease was compared in diabetics and non-diabetics, photographic studies of retinopathy, dietetic enquiries, studies of clotting factors, lipoprotein patterns and the like. The non-core investigations performed are listed in Appendix 2, and have been or will be reported separately. Centres are identified throughout this and other papers by the three-letter codes listed in Appendix 1 and Table 4.

\section{Materials and methods}

\section{Data collection and laboratory procedures}

During the period 1975 to 1977 a standard medical history and predetermined physical examination and investigation were recorded for all participants. The questionnaire, examination and investigation protocols are reproduced elsewhere $[3,4]$ but copies may be obtained on application to WHO. Variables recorded included age at interview and age at diagnosis of diabetes, characteristics of the diabetic state, its mode of presentation, the nature of treatment and history of vascular disease. Standard questions were used by the interviewing clinicians, and in almost all cases the information was acquired during a clinic visit earmarked for the collection of study data.

Following standard enquiry, patients were grouped into cigarettesmoking classes. Those who had never smoked, had ceased smoking, were currently smoking 1-14 cigarettes a day, 15-24 cigarettes/day and 25 or more cigarettes/day were allocated to classes $1-5$ respectively.

Height was measured without shoes, and weight while wearing indoor clothing. Body mass index (BMI) was computed by dividing the weight in kilograms by the square of the height in meters. Arterial 
Table 11. Mean body mass index $\left(\mathrm{kg} / \mathrm{m}^{2}\right)$ by age group, sex and centre

\begin{tabular}{|c|c|c|c|c|c|c|c|c|c|}
\hline \multirow[t]{3}{*}{ Centre } & \multirow[t]{3}{*}{ Sex } & \multicolumn{8}{|c|}{ Age groups (years) } \\
\hline & & \multicolumn{2}{|l|}{$35-41$} & \multicolumn{2}{|l|}{$42-48$} & \multicolumn{2}{|l|}{$49-54$} & \multicolumn{2}{|c|}{ All ages } \\
\hline & & Mean & $\mathrm{SD}$ & Mean & SD & Mean & $\mathrm{SD}$ & Mean & SD \\
\hline LON & $\begin{array}{l}\text { Male } \\
\text { Female }\end{array}$ & $\begin{array}{l}23.7 \\
25.5\end{array}$ & $\begin{array}{l}2.7 \\
4.6\end{array}$ & $\begin{array}{l}25.8 \\
26.8\end{array}$ & $\begin{array}{l}3.8 \\
4.8\end{array}$ & $\begin{array}{l}25.1 \\
26.2\end{array}$ & $\begin{array}{l}3.4 \\
4.6\end{array}$ & $\begin{array}{l}24.9 \\
26.3\end{array}$ & $\begin{array}{l}3.4 \\
4.7\end{array}$ \\
\hline SWI & $\begin{array}{l}\text { Male } \\
\text { Female }\end{array}$ & $\begin{array}{l}24.1 \\
24.5\end{array}$ & $\begin{array}{l}3.9 \\
5.2\end{array}$ & $\begin{array}{l}25.8 \\
27.3\end{array}$ & $\begin{array}{l}4.2 \\
6.5\end{array}$ & $\begin{array}{l}26.8 \\
28.3\end{array}$ & $\begin{array}{l}4.7 \\
5.4\end{array}$ & $\begin{array}{l}25.6 \\
26.6\end{array}$ & $\begin{array}{l}4.4 \\
6.0\end{array}$ \\
\hline BRU & $\begin{array}{l}\text { Male } \\
\text { Female }\end{array}$ & $\begin{array}{l}24.3 \\
24.0\end{array}$ & $\begin{array}{l}2.4 \\
4.0\end{array}$ & $\begin{array}{l}25.1 \\
25.4\end{array}$ & $\begin{array}{l}4.1 \\
4.1\end{array}$ & $\begin{array}{l}26.0 \\
27.1\end{array}$ & $\begin{array}{l}3.5 \\
5.5\end{array}$ & $\begin{array}{l}25.3 \\
25.6\end{array}$ & $\begin{array}{l}3.5 \\
4.8\end{array}$ \\
\hline MOS & $\begin{array}{l}\text { Male } \\
\text { Female }\end{array}$ & $\begin{array}{l}25.8 \\
29.6\end{array}$ & $\begin{array}{l}3.6 \\
5.8\end{array}$ & $\begin{array}{l}28.3 \\
30.7\end{array}$ & $\begin{array}{l}4.7 \\
5.7\end{array}$ & $\begin{array}{l}27.5 \\
30.5\end{array}$ & $\begin{array}{l}3.6 \\
4.9\end{array}$ & $\begin{array}{l}27.4 \\
30.3\end{array}$ & $\begin{array}{l}4.1 \\
5.4\end{array}$ \\
\hline WAR & $\begin{array}{l}\text { Male } \\
\text { Female }\end{array}$ & $\begin{array}{l}24.7 \\
27.2\end{array}$ & $\begin{array}{l}3.2 \\
5.4\end{array}$ & $\begin{array}{l}25.6 \\
30.1\end{array}$ & $\begin{array}{l}3.8 \\
5.0\end{array}$ & $\begin{array}{l}28.1 \\
30.1\end{array}$ & $\begin{array}{l}4.2 \\
5.7\end{array}$ & $\begin{array}{l}26.5 \\
29.5\end{array}$ & $\begin{array}{l}4.1 \\
5.8\end{array}$ \\
\hline BER & $\begin{array}{l}\text { Male } \\
\text { Female }\end{array}$ & $\begin{array}{l}25.1 \\
25.5\end{array}$ & $\begin{array}{l}4.3 \\
4.8\end{array}$ & $\begin{array}{l}25.4 \\
27.1\end{array}$ & $\begin{array}{l}3.6 \\
5.2\end{array}$ & $\begin{array}{l}26.7 \\
28.0\end{array}$ & $\begin{array}{l}3.5 \\
4.5\end{array}$ & $\begin{array}{l}25.8 \\
27.1\end{array}$ & $\begin{array}{l}3.8 \\
4.9\end{array}$ \\
\hline$Z A G$ & $\begin{array}{l}\text { Male } \\
\text { Female }\end{array}$ & $\begin{array}{l}25.4 \\
26.0\end{array}$ & $\begin{array}{l}2.9 \\
4.1\end{array}$ & $\begin{array}{l}25.7 \\
28.1\end{array}$ & $\begin{array}{l}3.2 \\
3.6\end{array}$ & $\begin{array}{l}26.8 \\
28.0\end{array}$ & $\begin{array}{l}3.1 \\
4.1\end{array}$ & $\begin{array}{l}26.0 \\
27.6\end{array}$ & $\begin{array}{l}3.1 \\
4.0\end{array}$ \\
\hline DEL & $\begin{array}{l}\text { Male } \\
\text { Female }\end{array}$ & $\begin{array}{l}22.6 \\
25.6\end{array}$ & $\begin{array}{l}4.2 \\
5.6\end{array}$ & $\begin{array}{l}24.2 \\
27.2\end{array}$ & $\begin{array}{l}4.1 \\
4.6\end{array}$ & $\begin{array}{l}23.7 \\
26.5\end{array}$ & $\begin{array}{l}3.3 \\
4.1\end{array}$ & $\begin{array}{l}23.5 \\
26.5\end{array}$ & $\begin{array}{l}3.9 \\
4.7\end{array}$ \\
\hline $\mathrm{HON}$ & $\begin{array}{l}\text { Male } \\
\text { Female }\end{array}$ & $\begin{array}{l}22.1 \\
24.3\end{array}$ & $\begin{array}{l}3.0 \\
4.0\end{array}$ & $\begin{array}{l}23.0 \\
25.1\end{array}$ & $\begin{array}{l}2.8 \\
4.2\end{array}$ & $\begin{array}{l}24.1 \\
25.0\end{array}$ & $\begin{array}{l}3.5 \\
3.9\end{array}$ & $\begin{array}{l}23.5 \\
24.8\end{array}$ & $\begin{array}{l}3.3 \\
4.0\end{array}$ \\
\hline TOK & $\begin{array}{l}\text { Male } \\
\text { Female }\end{array}$ & $\begin{array}{l}22.3 \\
21.9\end{array}$ & $\begin{array}{l}2.9 \\
3.7\end{array}$ & $\begin{array}{l}21.9 \\
22.3\end{array}$ & $\begin{array}{l}3.3 \\
2.9\end{array}$ & $\begin{array}{l}22.5 \\
23.2\end{array}$ & $\begin{array}{l}3.0 \\
3.3\end{array}$ & $\begin{array}{l}22.3 \\
22.6\end{array}$ & $\begin{array}{l}3.4 \\
3.4\end{array}$ \\
\hline HAV & $\begin{array}{l}\text { Male } \\
\text { Female }\end{array}$ & $\begin{array}{l}25.8 \\
28.1\end{array}$ & $\begin{array}{l}5.2 \\
6.2\end{array}$ & $\begin{array}{l}26.5 \\
27.9\end{array}$ & $\begin{array}{l}5.0 \\
6.4\end{array}$ & $\begin{array}{l}25.8 \\
27.5\end{array}$ & $\begin{array}{l}4.3 \\
5.5\end{array}$ & $\begin{array}{l}26.1 \\
27.8\end{array}$ & $\begin{array}{l}4.8 \\
6.1\end{array}$ \\
\hline OKL & $\begin{array}{l}\text { Male } \\
\text { Female }\end{array}$ & $\begin{array}{l}32.5 \\
34.0\end{array}$ & $\begin{array}{l}6.8 \\
8.3\end{array}$ & $\begin{array}{l}31.5 \\
33.1\end{array}$ & $\begin{array}{l}5.6 \\
6.9\end{array}$ & $\begin{array}{l}31.1 \\
33.3\end{array}$ & $\begin{array}{l}5.2 \\
7.9\end{array}$ & $\begin{array}{l}31.6 \\
33.4\end{array}$ & $\begin{array}{l}5.8 \\
7.6\end{array}$ \\
\hline ARI & $\begin{array}{l}\text { Male } \\
\text { Female }\end{array}$ & $\begin{array}{l}30.6 \\
32.8\end{array}$ & $\begin{array}{l}8.7 \\
6.1\end{array}$ & $\begin{array}{l}28.5 \\
33.1\end{array}$ & $\begin{array}{l}5.2 \\
6.4\end{array}$ & $\begin{array}{l}28.4 \\
31.2\end{array}$ & $\begin{array}{l}3.8 \\
6.1\end{array}$ & $\begin{array}{l}29.1 \\
32.2\end{array}$ & $\begin{array}{l}6.0 \\
6.2\end{array}$ \\
\hline BUL & $\begin{array}{l}\text { Male } \\
\text { Female }\end{array}$ & $\begin{array}{l}24.7 \\
25.2\end{array}$ & $\begin{array}{l}3.1 \\
4.3\end{array}$ & $\begin{array}{l}26.3 \\
28.0\end{array}$ & $\begin{array}{l}3.5 \\
5.1\end{array}$ & $\begin{array}{l}26.7 \\
27.8\end{array}$ & $\begin{array}{l}3.6 \\
5.4\end{array}$ & $\begin{array}{l}26.1 \\
27.2\end{array}$ & $\begin{array}{l}3.5 \\
5.1\end{array}$ \\
\hline Total & $\begin{array}{l}\text { Male } \\
\text { Female }\end{array}$ & $\begin{array}{l}25.1 \\
26.8\end{array}$ & $\begin{array}{l}4.9 \\
6.3\end{array}$ & $\begin{array}{l}25.9 \\
28.4\end{array}$ & $\begin{array}{l}4.7 \\
6.1\end{array}$ & $\begin{array}{l}26.3 \\
28.1\end{array}$ & $\begin{array}{l}4.3 \\
5.9\end{array}$ & $\begin{array}{l}25.6 \\
27.9\end{array}$ & $\begin{array}{l}4.7 \\
6.1\end{array}$ \\
\hline
\end{tabular}

blood pressure was measured with a standard clinical mercury sphygmomanometer and a $10 \times 22-\mathrm{cm}$ cuff, after at least $10 \mathrm{~min}$ in the sitting position, and after $30 \mathrm{~min}$ or more without smoking or eating. Diastolic pressure was recorded both at the time of sudden muffling of sounds (Korotkov phase 4), and at complete disappearance of sounds (Korotkov phase 5). The WHO definition of hypertension was used (systolic blood pressure $160 \mathrm{mmHg}$ or more, or a phase 5 diastolic blood pressure $95 \mathrm{mmHg}$ or more, or receiving treatment with antihypertensive drugs.

The fundi were examined by direct ophthalmoscopy of each eye through dilated pupils, standardizing the observation to a timed twominute period for each eye. Prior to the study all participating centres took part in a workshop where a number of selected patients, including some with normal fundi, were examined using standardized methods by all participants. Their results were then analysed and discussed. A set of standard colour photographs of the lesions sought was supplied to each examining centre in a further attempt to standardize the recognition and documentation of the various lesions.

Venous blood with heparin anticoagulant was collected for cholesterol and creatinine determinations. The measurements were made both locally and at one of two WHO-collaborating lipid centres in Atlanta (Georgia, USA) and Moscow, USSR, using accepted techniques [5]. The core protocol did not require measurement of cholesterol subfractions; uniform techniques and quality-control monitoring for these measurements through the WHO collaborating centres were not available at the time. Some of the participating centres also made detailed lipid and other studies (non-core) on their samples and they are listed in Appendix 2.

A fresh specimen of urine was collected and tested by a standard sulphosalicylic solution method by all centres, except Zagreb where the Albustix method was used [6]. The intensity of protein precipitation was recorded and used in analyses as an indicator of renal disease severity. Positive tests were graded from 1 (barely perceptible turbidity) to 4 (dense flocculent precipitate).

\section{Large-vessel disease endpoints}

Cardiovascular questionnaire responses and Minnesota codes for the ECGs were used in two ways, either as individual items of abnormality (components) or grouped in various combinations (composites) (Table 2).

Components. From the standard questionnaire responses, angina pectoris, history of possible infarction and intermittent claudication were identified as recommended by Blackburn and Rose [7]. The categories 'amputation or gangrene' and 'stroke' were determined from responses to questions specially formulated for this study and included in the questionnaire: 
Table 12. Prevalence of cigarette smoking (\%) by sex and centre

\begin{tabular}{|c|c|c|c|c|c|c|}
\hline \multirow[t]{2}{*}{ Centre } & \multirow[t]{2}{*}{ Sex } & \multicolumn{5}{|c|}{ Cigarette use } \\
\hline & & $\begin{array}{l}>24 \\
\text { Daily }\end{array}$ & $\begin{array}{l}15-24 \\
\text { Daily }\end{array}$ & $\begin{array}{l}1-14 \\
\text { Daily }\end{array}$ & Ex-smoker & $\begin{array}{l}\text { Never } \\
\text { Smoked }\end{array}$ \\
\hline LON & $\begin{array}{l}\text { Male } \\
\text { Female }\end{array}$ & $\begin{array}{r}11.8 \\
9.1\end{array}$ & $\begin{array}{l}17.7 \\
11.9\end{array}$ & $\begin{array}{l}11.4 \\
13.2\end{array}$ & $\begin{array}{l}32.7 \\
22.6\end{array}$ & $\begin{array}{l}26.4 \\
43.2\end{array}$ \\
\hline SWI & $\begin{array}{l}\text { Male } \\
\text { Female }\end{array}$ & $\begin{array}{l}8.6 \\
0.8\end{array}$ & $\begin{array}{r}13.3 \\
5.1\end{array}$ & $\begin{array}{l}16.9 \\
21.5\end{array}$ & $\begin{array}{l}34.9 \\
16.0\end{array}$ & $\begin{array}{l}26.3 \\
56.6\end{array}$ \\
\hline BRU & $\begin{array}{l}\text { Male } \\
\text { Female }\end{array}$ & $\begin{array}{r}15.3 \\
5.9\end{array}$ & $\begin{array}{r}18.6 \\
5.9\end{array}$ & $\begin{array}{r}20.8 \\
9.1\end{array}$ & $\begin{array}{r}21.2 \\
5.9\end{array}$ & $\begin{array}{l}24.2 \\
73.1\end{array}$ \\
\hline MOS & $\begin{array}{l}\text { Male } \\
\text { Female }\end{array}$ & $\begin{array}{l}6.2 \\
0.4\end{array}$ & $\begin{array}{r}19.0 \\
2.2\end{array}$ & $\begin{array}{r}10.6 \\
5.1\end{array}$ & $\begin{array}{r}26.1 \\
1.8\end{array}$ & $\begin{array}{l}38.1 \\
90.5\end{array}$ \\
\hline WAR & $\begin{array}{l}\text { Male } \\
\text { Female }\end{array}$ & $\begin{array}{r}12.9 \\
2.4\end{array}$ & $\begin{array}{r}25.3 \\
6.1\end{array}$ & $\begin{array}{l}13.3 \\
12.2\end{array}$ & $\begin{array}{l}25.3 \\
15.1\end{array}$ & $\begin{array}{l}23.2 \\
64.1\end{array}$ \\
\hline BER & $\begin{array}{l}\text { Male } \\
\text { Female }\end{array}$ & $\begin{array}{l}5.6 \\
0.4\end{array}$ & $\begin{array}{r}16.8 \\
6.2\end{array}$ & $\begin{array}{l}21.1 \\
21.1\end{array}$ & $\begin{array}{l}36.8 \\
14.2\end{array}$ & $\begin{array}{l}19.6 \\
58.2\end{array}$ \\
\hline $\mathrm{ZAG}$ & $\begin{array}{l}\text { Male } \\
\text { Female }\end{array}$ & $\begin{array}{r}12.2 \\
1.1\end{array}$ & $\begin{array}{r}26.6 \\
3.9\end{array}$ & $\begin{array}{l}6.3 \\
6.7\end{array}$ & $\begin{array}{r}22.5 \\
4.4\end{array}$ & $\begin{array}{l}32.4 \\
83.9\end{array}$ \\
\hline DEL & $\begin{array}{l}\text { Male } \\
\text { Female }\end{array}$ & $\begin{array}{l}2.4 \\
0\end{array}$ & $\begin{array}{c}10.0 \\
0\end{array}$ & $\begin{array}{r}20.4 \\
0.4\end{array}$ & $\begin{array}{r}18.7 \\
1.1\end{array}$ & $\begin{array}{l}48.4 \\
98.5\end{array}$ \\
\hline $\mathrm{HON}$ & $\begin{array}{l}\text { Male } \\
\text { Female }\end{array}$ & $\begin{array}{l}8.1 \\
0\end{array}$ & $\begin{array}{r}22.7 \\
3.1\end{array}$ & $\begin{array}{l}26.3 \\
11.2\end{array}$ & $\begin{array}{r}10.6 \\
4.5\end{array}$ & $\begin{array}{l}32.3 \\
81.3\end{array}$ \\
\hline TOK & $\begin{array}{l}\text { Male } \\
\text { Female }\end{array}$ & $\begin{array}{r}25.5 \\
1.5\end{array}$ & $\begin{array}{r}23.8 \\
6.0\end{array}$ & $\begin{array}{l}15.3 \\
10.4\end{array}$ & $\begin{array}{r}19.1 \\
4.0\end{array}$ & $\begin{array}{l}16.2 \\
78.1\end{array}$ \\
\hline HAV & $\begin{array}{l}\text { Male } \\
\text { Female }\end{array}$ & $\begin{array}{l}9.7 \\
2.7\end{array}$ & $\begin{array}{r}20.9 \\
8.9\end{array}$ & $\begin{array}{l}40.7 \\
21.4\end{array}$ & $\begin{array}{l}8.1 \\
8.9\end{array}$ & $\begin{array}{l}20.5 \\
58.0\end{array}$ \\
\hline OKL & $\begin{array}{l}\text { Male } \\
\text { Female }\end{array}$ & $\begin{array}{l}5.9 \\
2.3\end{array}$ & $\begin{array}{r}13.0 \\
7.6\end{array}$ & $\begin{array}{l}32.6 \\
25.1\end{array}$ & $\begin{array}{l}27.0 \\
25.6\end{array}$ & $\begin{array}{l}21.5 \\
39.4\end{array}$ \\
\hline ARI & $\begin{array}{l}\text { Male } \\
\text { Female }\end{array}$ & $\begin{array}{l}0 \\
0\end{array}$ & $\begin{array}{l}1.1 \\
0.6\end{array}$ & $\begin{array}{l}43.7 \\
22.7\end{array}$ & $\begin{array}{l}42.5 \\
38.3\end{array}$ & $\begin{array}{l}12.6 \\
38.3\end{array}$ \\
\hline BUL & $\begin{array}{l}\text { Male } \\
\text { Female }\end{array}$ & $\begin{array}{l}5.0 \\
0\end{array}$ & $\begin{array}{r}18.5 \\
0.8\end{array}$ & $\begin{array}{r}13.1 \\
4.4\end{array}$ & $\begin{array}{r}30.2 \\
5.6\end{array}$ & $\begin{array}{l}33.3 \\
89.2\end{array}$ \\
\hline
\end{tabular}

'Angina pectoris' includes both 'on the flat' and 'uphill only' grades of response. 'Possible infarction' was recorded as positive after an affirmative answer to the single question about central chest pain lasting half an hour or more. 'Intermittent claudication', like 'angina', includes both grades of response ('on the flat' and 'uphill only'). 'Amputation or gangrene' was recorded if there was necrotic or surgical loss of tissue in the toe, foot or leg due, in the judgement of the investigator, to arterial obstruction rather than neuropathic damage. 'Stroke' was recorded only when there was a history of persistent $(>24 \mathrm{~h})$ hemiplegia.

Standard 12-lead electrocardiograms were recorded on $98.8 \%$ of the study participants. The same two experienced readers analysed all the tracings using the Minnesota Code, and a third reader arbitrated disagreements (less than $0.5 \%$ of readings) [8]. The following LargeVessel Disease component items were derived from the ECG Code results:

'ECG Coronary Probable' consisting of Codes 1.1 and 1.2 (large Q' QS waves), and Code 7.1 (complete left bundle branch block).

'ECG Coronary Possible' consisting of Code 1.3 (small Q waves), Code 4.1, 4.2 and 4.3 (S-T segment abnormalities) and Codes 5.1, 5.2 and 5.3 (T wave abnormalities). 'ECG Coronary Unlikely' comprising all other ECG records whether coded as normal or abnormal.

Composites. The component indicators of large vessel disease were grouped in a variety of ways in order to express either particular regions of the circulation affected or degrees of severity of arterial disease (Table 1).

'Heart Vascular Disease' (HVD) includes subjects positive for 'ECG Probable', 'ECG Possible', 'Angina Pectoris' or 'Possible Infarction' alone or in combination.

'Leg Vascular Disease' (Leg VD) combines subjects positive for either 'Intermittent claudication' or 'Amputation'.

'Large-Vessel Disease' (LVD) is an inclusive category which comprises individuals with any or all of the components of macrovascular disease.

LVD is divided into two 'severity' sublasses:

'LVD Severe' consists of the most clearly verifiable LVD endpoints only, i. e. 'ECG Coronary Probable' or 'Amputation'.

'LVD Intermediate' includes patients with 'Angina Pectoris', 'Possible Infarction', 'Intermittent Claudication', 'Stroke' or 'ECG Coronary Possible'.

Table 13. Distribution of total plasma cholesterol levels (\%) by sex and centre

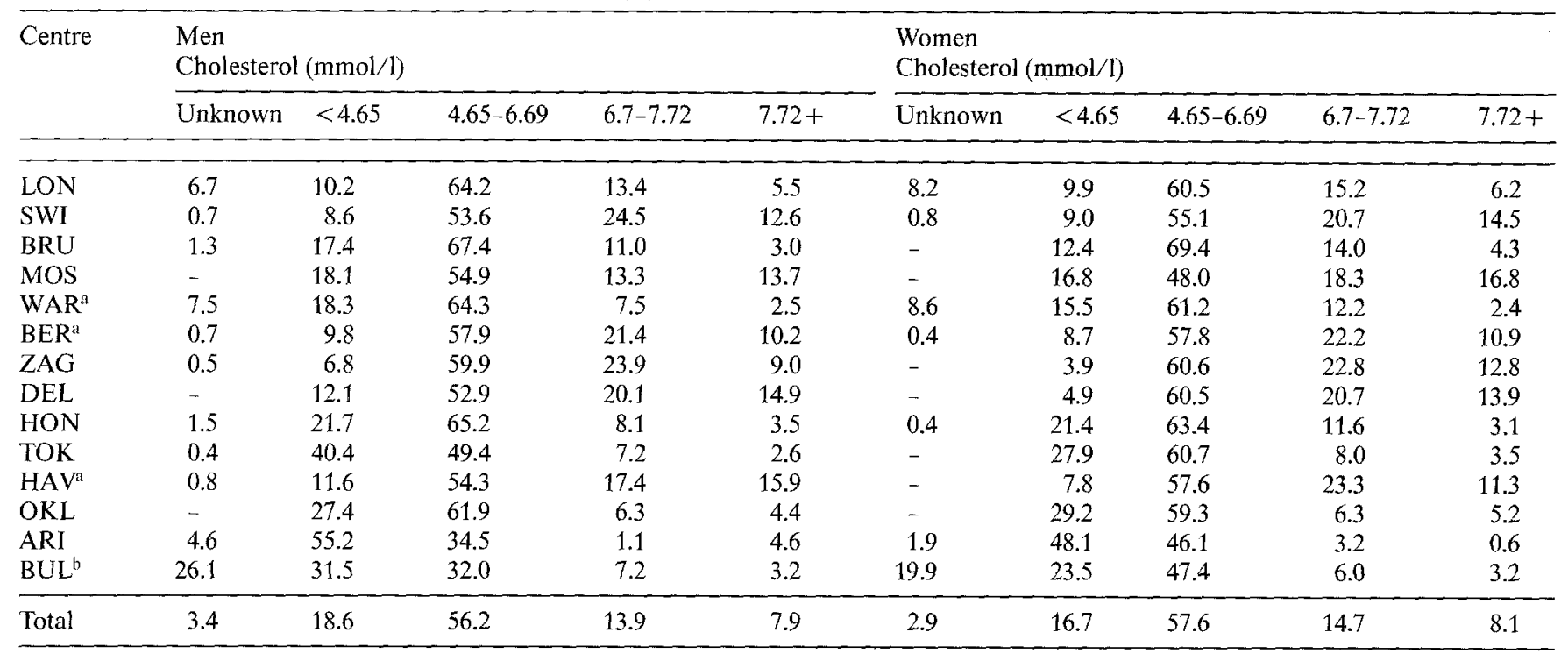

${ }^{a}$ Measured in local laboratory; ${ }^{b}$ measured in London laboratory 
Table 14. Mean total cholesterol $(\mathrm{mmol} / \mathrm{l})$ by age group, sex and centre

\begin{tabular}{|c|c|c|c|c|c|c|c|c|c|}
\hline \multirow[t]{3}{*}{ Centre } & \multirow[t]{3}{*}{ Sex } & \multicolumn{8}{|c|}{ Age groups (years) } \\
\hline & & \multicolumn{2}{|l|}{$35-41$} & \multicolumn{2}{|l|}{$42-48$} & \multicolumn{2}{|l|}{$49-54$} & \multicolumn{2}{|c|}{ All ages } \\
\hline & & Mean & SD & $\overline{\text { Mean }}$ & $\mathrm{SD}$ & Mean & SD & Mean & SD \\
\hline LON & $\begin{array}{l}\text { Male } \\
\text { Female }\end{array}$ & $\begin{array}{l}5.8 \\
5.8\end{array}$ & $\begin{array}{l}1.1 \\
1.0\end{array}$ & $\begin{array}{l}6.0 \\
5.9\end{array}$ & $\begin{array}{l}1.0 \\
1.2\end{array}$ & $\begin{array}{l}5.8 \\
6.0\end{array}$ & $\begin{array}{l}1.2 \\
1.2\end{array}$ & $\begin{array}{l}5.9 \\
5.9\end{array}$ & $\begin{array}{l}1.2 \\
1.2\end{array}$ \\
\hline SWI & $\begin{array}{l}\text { Male } \\
\text { Female }\end{array}$ & $\begin{array}{l}6.1 \\
6.0\end{array}$ & $\begin{array}{l}1.4 \\
1.2\end{array}$ & $\begin{array}{l}6.3 \\
6.4\end{array}$ & $\begin{array}{l}1.5 \\
1.5\end{array}$ & $\begin{array}{l}6.8 \\
6.7\end{array}$ & $\begin{array}{l}1.4 \\
1.3\end{array}$ & $\begin{array}{l}6.4 \\
6.4\end{array}$ & $\begin{array}{l}1.5 \\
1.4\end{array}$ \\
\hline BRU & $\begin{array}{l}\text { Male } \\
\text { Female }\end{array}$ & $\begin{array}{l}5.5 \\
5.3\end{array}$ & $\begin{array}{l}1.4 \\
0.9\end{array}$ & $\begin{array}{l}5.6 \\
5.7\end{array}$ & $\begin{array}{l}0.9 \\
1.1\end{array}$ & $\begin{array}{l}5.5 \\
6.1\end{array}$ & $\begin{array}{l}0.9 \\
1.3\end{array}$ & $\begin{array}{l}5.5 \\
5.7\end{array}$ & $\begin{array}{l}1.1 \\
1.2\end{array}$ \\
\hline MOS & $\begin{array}{l}\text { Male } \\
\text { Female }\end{array}$ & $\begin{array}{l}5.8 \\
6.2\end{array}$ & $\begin{array}{l}1.9 \\
2.2\end{array}$ & $\begin{array}{l}6.3 \\
6.5\end{array}$ & $\begin{array}{l}2.1 \\
2.2\end{array}$ & $\begin{array}{l}6.0 \\
6.5\end{array}$ & $\begin{array}{l}1.4 \\
1.9\end{array}$ & $\begin{array}{l}6.1 \\
6.4\end{array}$ & $\begin{array}{l}1.8 \\
2.1\end{array}$ \\
\hline WAR $^{\mathrm{a}}$ & $\begin{array}{l}\text { Male } \\
\text { Female }\end{array}$ & $\begin{array}{l}5.3 \\
5.2\end{array}$ & $\begin{array}{l}0.9 \\
1.1\end{array}$ & $\begin{array}{l}5.7 \\
5.4\end{array}$ & $\begin{array}{l}1.3 \\
1.0\end{array}$ & $\begin{array}{l}5.4 \\
5.9\end{array}$ & $\begin{array}{l}1.2 \\
1.2\end{array}$ & $\begin{array}{l}5.5 \\
5.6\end{array}$ & $\begin{array}{l}1.2 \\
1.1\end{array}$ \\
\hline $\mathrm{BER}^{\mathrm{a}}$ & $\begin{array}{l}\text { Male } \\
\text { Female }\end{array}$ & $\begin{array}{l}6.1 \\
6.2\end{array}$ & $\begin{array}{l}1.3 \\
2.0\end{array}$ & $\begin{array}{l}6.3 \\
6.1\end{array}$ & $\begin{array}{l}1.3 \\
1.2\end{array}$ & $\begin{array}{l}6.3 \\
6.6\end{array}$ & $\begin{array}{l}1.8 \\
1.7\end{array}$ & $\begin{array}{l}6.3 \\
6.3\end{array}$ & $\begin{array}{l}1.5 \\
1.6\end{array}$ \\
\hline $\mathrm{ZAG}$ & $\begin{array}{l}\text { Male } \\
\text { Female }\end{array}$ & $\begin{array}{l}6.3 \\
6.3\end{array}$ & $\begin{array}{l}1.1 \\
1.7\end{array}$ & $\begin{array}{l}6.4 \\
6.5\end{array}$ & $\begin{array}{l}1.5 \\
1.6\end{array}$ & $\begin{array}{l}6.4 \\
6.9\end{array}$ & $\begin{array}{l}1.3 \\
2.1\end{array}$ & $\begin{array}{l}6.4 \\
6.6\end{array}$ & $\begin{array}{l}1.3 \\
1.9\end{array}$ \\
\hline DEL & $\begin{array}{l}\text { Male } \\
\text { Female }\end{array}$ & $\begin{array}{l}6.5 \\
6.2\end{array}$ & $\begin{array}{l}2.3 \\
1.2\end{array}$ & $\begin{array}{l}6.5 \\
6.1\end{array}$ & $\begin{array}{l}2.1 \\
1.2\end{array}$ & $\begin{array}{l}6.1 \\
6.6\end{array}$ & $\begin{array}{l}1.3 \\
1.4\end{array}$ & $\begin{array}{l}6.3 \\
6.3\end{array}$ & $\begin{array}{l}1.9 \\
1.3\end{array}$ \\
\hline HON & $\begin{array}{l}\text { Male } \\
\text { Female }\end{array}$ & $\begin{array}{l}5.9 \\
5.4\end{array}$ & $\begin{array}{l}0.9 \\
1.1\end{array}$ & $\begin{array}{l}5.5 \\
5.5\end{array}$ & $\begin{array}{l}1.7 \\
1.2\end{array}$ & $\begin{array}{l}5.5 \\
5.7\end{array}$ & $\begin{array}{l}1.1 \\
1.2\end{array}$ & $\begin{array}{l}5.5 \\
5.6\end{array}$ & $\begin{array}{l}1.3 \\
1.2\end{array}$ \\
\hline TOK & $\begin{array}{l}\text { Male } \\
\text { Female }\end{array}$ & $\begin{array}{l}5.0 \\
5.0\end{array}$ & $\begin{array}{l}1.0 \\
1.1\end{array}$ & $\begin{array}{l}5.1 \\
5.2\end{array}$ & $\begin{array}{l}1.2 \\
1.0\end{array}$ & $\begin{array}{l}5.1 \\
5.5\end{array}$ & $\begin{array}{l}1.1 \\
1.3\end{array}$ & $\begin{array}{l}5.1 \\
5.3\end{array}$ & $\begin{array}{l}1.1 \\
1.2\end{array}$ \\
\hline $\mathrm{HAV}^{\mathrm{a}}$ & $\begin{array}{l}\text { Male } \\
\text { Female }\end{array}$ & $\begin{array}{l}6.3 \\
5.8\end{array}$ & $\begin{array}{l}1.5 \\
1.2\end{array}$ & $\begin{array}{l}6.3 \\
6.2\end{array}$ & $\begin{array}{l}2.0 \\
1.2\end{array}$ & $\begin{array}{l}6.4 \\
6.8\end{array}$ & $\begin{array}{l}1.5 \\
1.4\end{array}$ & $\begin{array}{l}6.4 \\
6.3\end{array}$ & $\begin{array}{l}1.7 \\
1.3\end{array}$ \\
\hline OKL & $\begin{array}{l}\text { Male } \\
\text { Female }\end{array}$ & $\begin{array}{l}5.2 \\
5.3\end{array}$ & $\begin{array}{l}1.0 \\
1.9\end{array}$ & $\begin{array}{l}5.6 \\
5.4\end{array}$ & $\begin{array}{l}1.6 \\
1.1\end{array}$ & $\begin{array}{l}5.5 \\
5.4\end{array}$ & $\begin{array}{l}2.3 \\
1.5\end{array}$ & $\begin{array}{l}5.5 \\
5.4\end{array}$ & $\begin{array}{l}1.8 \\
1.5\end{array}$ \\
\hline ARI & $\begin{array}{l}\text { Male } \\
\text { Female }\end{array}$ & $\begin{array}{l}4.9 \\
4.7\end{array}$ & $\begin{array}{l}1.3 \\
0.9\end{array}$ & $\begin{array}{l}4.9 \\
4.6\end{array}$ & $\begin{array}{l}2.3 \\
0.9\end{array}$ & $\begin{array}{l}4.6 \\
4.8\end{array}$ & $\begin{array}{l}1.2 \\
1.0\end{array}$ & $\begin{array}{l}4.8 \\
4.7\end{array}$ & $\begin{array}{l}1.6 \\
0.9\end{array}$ \\
\hline $\mathrm{BUL}^{\mathrm{b}}$ & $\begin{array}{l}\text { Male } \\
\text { Female }\end{array}$ & $\begin{array}{l}5.1 \\
5.1\end{array}$ & $\begin{array}{l}1.5 \\
1.1\end{array}$ & $\begin{array}{l}4.7 \\
5.4\end{array}$ & $\begin{array}{l}1.1 \\
1.4\end{array}$ & $\begin{array}{l}5.3 \\
5.4\end{array}$ & $\begin{array}{l}1.3 \\
1.1\end{array}$ & $\begin{array}{l}5.1 \\
5.4\end{array}$ & $\begin{array}{l}1.3 \\
1.2\end{array}$ \\
\hline Total & $\begin{array}{l}\text { Male } \\
\text { Female }\end{array}$ & $\begin{array}{l}5.8 \\
5.7\end{array}$ & $\begin{array}{l}1.5 \\
1.5\end{array}$ & $\begin{array}{l}5.9 \\
5.8\end{array}$ & $\begin{array}{l}1.7 \\
1.4\end{array}$ & $\begin{array}{l}5.8 \\
6.0\end{array}$ & $\begin{array}{l}1.5 \\
1.5\end{array}$ & $\begin{array}{l}5.8 \\
5.9\end{array}$ & $\begin{array}{l}1.6 \\
1.5\end{array}$ \\
\hline
\end{tabular}

${ }^{a}$ Measured in local laboratory; ${ }^{b}$ Measured in London laboratory

Two further classes were distinguished for analytical purposes, defined as follows:

'Stroke Only' includes patients with a history of hemiplegic stroke, but with no other evidence of LVD. 'LVD-Free' includes all patients without evidence of LVD, whether small-vessel disease (SVD) was present or not.

\section{Small-vessel disease endpoints}

As with LVD, small-vessel disease indices, derived exclusively from standardized examination of the eye and urine, were categorized either by component abnormalities or in composite groupings.

Components. Eight components of retinopathy were derived from the examination findings and are defined as follows:

'Retinopathic Blindness' was the class to which blind or severely visually-handicapped patients were assigned when this disability was due to or associated with new-vessel formation, vitreous haemorrhage or opacity, large red lesions, or six or more hard exudates in one or both eyes. Blind patients with cataracts which obscured the view of both fundi were classified separately. 'New Vessels' were accepted only when recorded as 'definitely present' on fundal examination.
'Small Red Lesions' were microaneurysms and haemorrhagic spots less than the breadth of the largest retinal artery as it crossed the edge of the optic disc.

'Medium Red Lesions' were larger than the lesions described above, but less in their largest diameter than the breadth of the optic disc.

'Large Red Lesions' were haemorrhages with their largest diameter greater than that of the optic disc.

'Hard Exudates' were described, illustrated and distinguished from drüsen. Their extent was estimated as the number of 'disc-sized areas' affected.

'Soft Exudates' (or cotton-wool spots) were described and illustrated in the reference photographs. Their number was counted and recorded.

'Vitreous Opacity or Haemorrhage' described definite opacification or bleeding into the vitreous body.

Small-vessel disease of the kidney (SVDK) was defined by the presence of proteinuria using the standardized salicyl-sulphonic acid test:

'Severe Small-Vessel Disease of the Kidney' (SVDK Severe) - proteinuria (heavy or very heavy) with a flocculent or heavy precipitate. 
Table 15. Insulin use: proportions (\%) by diabetes duration, sex and centre

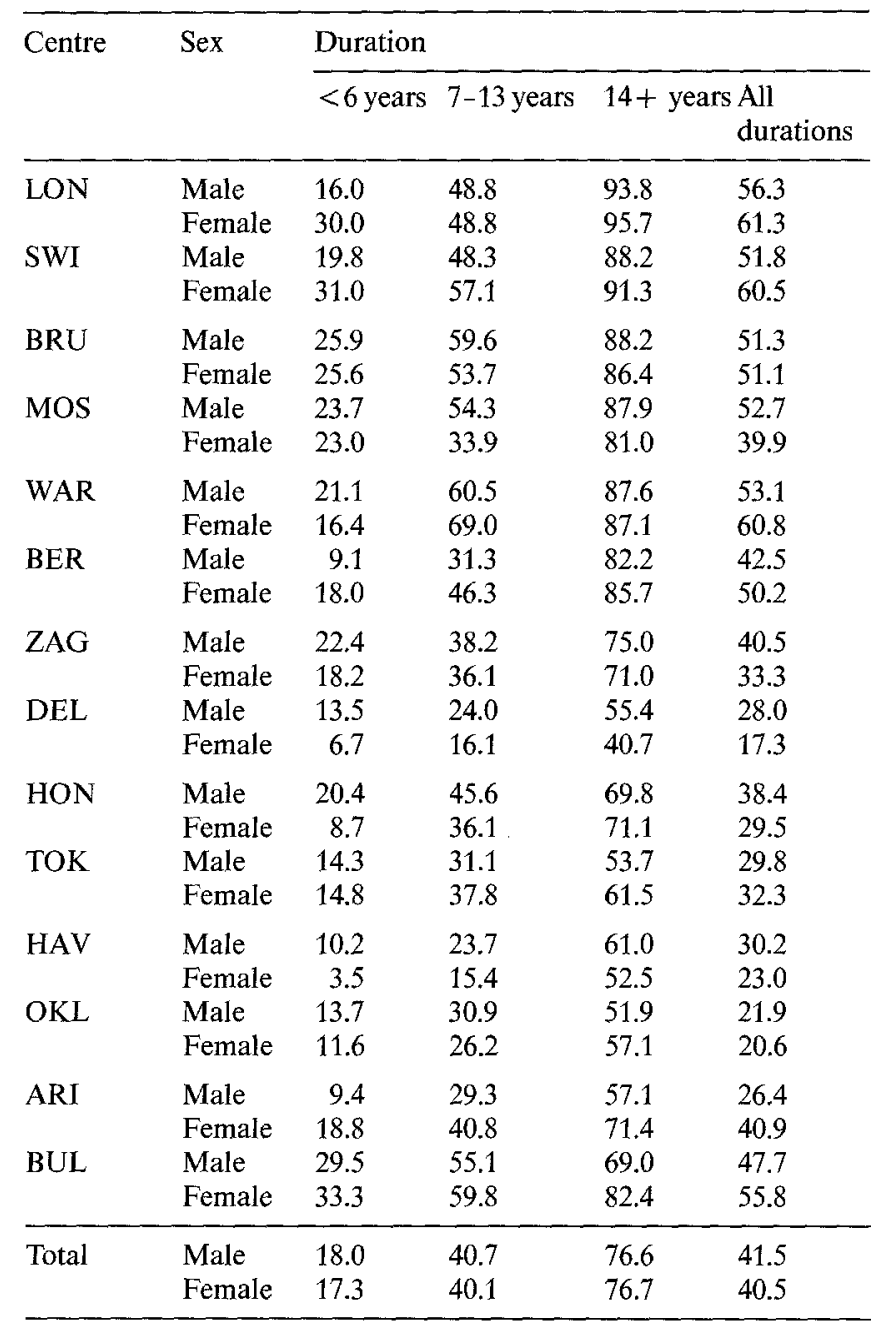

'Minimal Small-Vessel Disease of the Kidney' (SVDK Minimal) proteinuria with lesser degrees of turbidity than described for SVDK severe.

Composites. The small-vessel disease components were grouped into composites for comparative and analytic purposes. These are described below and are also shown schematically in Table 3 .

'Severe Small-Vessel Disease of the Eye' (SVDE Severe) includes

those with 'Retinopathic Blindness' in either eye, 'New Vessels' in either eye, 'Vitreous Opacity or Haemorrhage' in either eye or 'Large Red Lesions' in either eye.

'Medium Small-Vessel Disease of the eye' (SVDE Medium) includes those lacking the severe features, but with 'Small Red Lesions' (two or more) in either eye, 'Medium Red Lesions' (one or more) in either eye, or 'Hard or Soft Exudates' (two or more) in either eye.

'Minimal Small-Vessel Disease of the Eye' (SVDE Minimal) comprised a group lacking any of the abnormalities above and in which evidence of retinopathy was weak or doubtful.

It includes those with 'Small Red Lesions' (one lesion) in either eye, a single 'Hard or Soft Exudate' in either eye or 'New Vessels' coded as 'doubtful' in either eye.

Eye abnormalities and proteinuria were combined to form a further set of composites as follows (Table 3):

Small-Vessel Disease Severe = SVDE Severe + SVDK Severe.

Small-Vessel Disease Major = SVDE Severe or SVDK Severe plus any category of the other.
Table 16. Oral anti-diabetic drug treatment: proportions (\%) by diabetes duration, sex and centre

\begin{tabular}{|c|c|c|c|c|c|}
\hline \multirow[t]{2}{*}{ Centre } & \multirow[t]{2}{*}{ Sex } & \multicolumn{4}{|l|}{ Duration } \\
\hline & & $0-6$ years & $7-13$ years & $14+$ years & $\begin{array}{l}\text { All } \\
\text { durations }\end{array}$ \\
\hline \multirow[t]{2}{*}{ LON } & Male & 44.0 & 42.7 & 4.1 & 28.3 \\
\hline & Female & 45.7 & 47.5 & 4.3 & 30.5 \\
\hline \multirow[t]{2}{*}{ SWI } & Male & 58.3 & 44.9 & 11.8 & 38.5 \\
\hline & Female & 50.6 & 41.6 & 6.5 & 32.0 \\
\hline \multirow[t]{2}{*}{ BRU } & Male & 32.8 & 30.8 & 7.4 & 25.0 \\
\hline & Female & 32.6 & 31.7 & 10.2 & 26.3 \\
\hline \multirow[t]{2}{*}{ MOS } & Male & 48.7 & 40.2 & 10.3 & 35.4 \\
\hline & Female & 58.0 & 50.4 & 13.8 & 45.4 \\
\hline \multirow[t]{2}{*}{ WAR } & Male & 67.1 & 39.5 & 12.4 & 38.2 \\
\hline & Female & 64.4 & 29.6 & 9.9 & 31.8 \\
\hline \multirow[t]{2}{*}{ BER } & Male & 43.2 & 34.4 & 14.9 & 30.2 \\
\hline & Female & 47.2 & 25.3 & 6.6 & 26.2 \\
\hline \multirow[t]{2}{*}{$\mathrm{ZAG}$} & Male & 40.8 & 47.1 & 19.6 & 37.4 \\
\hline & Female & 52.3 & 47.5 & 19.4 & 45.0 \\
\hline \multirow[t]{2}{*}{ DEL } & Male & 66.7 & 74.0 & 41.9 & 63.0 \\
\hline & Female & 70.8 & 80.5 & 59.3 & 71.4 \\
\hline \multirow[t]{2}{*}{ HON } & Male & 75.5 & 54.4 & 30.2 & 59.6 \\
\hline & Female & 88.3 & 63.9 & 28.9 & 69.2 \\
\hline \multirow[t]{2}{*}{ TOK } & Male & 45.1 & 45.6 & 35.2 & 43.0 \\
\hline & Female & 56.8 & 48.6 & 33.3 & 49.3 \\
\hline \multirow[t]{2}{*}{ HAV } & Male & 62.5 & 58.1 & 37.7 & 53.5 \\
\hline & Female & 77.9 & 76.9 & 41.3 & 66.1 \\
\hline \multirow[t]{2}{*}{ OKL } & Male & 56.6 & 51.5 & 37.0 & 53.3 \\
\hline & Female & 53.6 & 53.6 & 32.7 & 50.9 \\
\hline \multirow[t]{2}{*}{ ARI } & Male & 25.0 & 34.1 & 21.4 & 28.7 \\
\hline & Female & 25.0 & 33.8 & 22.9 & 28.6 \\
\hline \multirow[t]{2}{*}{ BUL } & Male & 56.8 & 40.6 & 29.3 & 44.6 \\
\hline & Female & 55.2 & 36.8 & 14.7 & 37.8 \\
\hline \multirow[t]{2}{*}{ Total } & Male & 53.1 & 46.7 & 20.4 & 42.0 \\
\hline & Female & 57.0 & 48.8 & 19.3 & 44.4 \\
\hline
\end{tabular}

Small-Vessel Disease Medium = All or any categories of SVDE or SVDK apart from severe.

Small-Vessel-Disease-Free $=$ those with no evidence of SVDE or SVDK whether or not LVD was present.

\section{Statistical procedures}

Standard descriptive statistics were used in most of the analyses. Comparisons were made within each centre, between centres and, in some analyses, with data pooled from all centres. Relationships between the various analytical variables such as smoking, blood pressure and cholesterol level and the vascular disease endpoints were analysed by comparing means for affected and unaffected groups and by determining correlation coefficients. Multivariate techniques were used to assess the independent contribution of individual variables when these might be spuriously created or obscured by the confounding effects of other variables.

Simple direct standardization for age or known duration of diabetes was employed to allow comparison of centres with differing age or duration structures. The probability that differences between group means were due to chance was assessed by two-tailed Student's t-test. A standard general linear-model multiple regression program (SPSS System) was used with one dependent variable for analyses of the relation between independent variables with renal and retinal disease. For the large-vessel disease endpoints, specific independent variables were tested for an association with the Walker-Duncan logistic regression procedure using the maximum-likelihood method of estimation [9]. 
Table 17. Diet-only treatment: proportions (\%) by diabetes duration, sex and centre

\begin{tabular}{|c|c|c|c|c|c|}
\hline \multirow[t]{2}{*}{ Centre } & \multirow[t]{2}{*}{ Sex } & \multicolumn{4}{|l|}{ Duration } \\
\hline & & $0-6$ years & $7-13$ years & $14+$ years & $\begin{array}{l}\text { All } \\
\text { durations }\end{array}$ \\
\hline \multirow[t]{2}{*}{ LON } & Male & 40.0 & 8.5 & 2.1 & 15.4 \\
\hline & Female & 24.3 & 3.8 & - & 8.2 \\
\hline \multirow[t]{2}{*}{ SWI } & Male & 21.9 & 6.7 & - & 9.7 \\
\hline & Female & 18.4 & 1.3 & 2.2 & 7.4 \\
\hline \multirow[t]{2}{*}{ BRU } & Male & 41.4 & 9.6 & 4.4 & 23.7 \\
\hline & Female & 41.9 & 14.6 & 3.4 & 23.7 \\
\hline \multirow[t]{2}{*}{ MOS } & Male & 27.6 & 5.4 & 1.7 & 11.9 \\
\hline & Female & 19.0 & 15.7 & 5.2 & 14.7 \\
\hline \multirow[t]{2}{*}{ WAR } & Male & 11.8 & - & - & 3.7 \\
\hline & Female & 19.2 & 1.4 & 3.0 & 7.3 \\
\hline \multirow[t]{2}{*}{ BER } & Male & 47.7 & 34.4 & 3.0 & 27.4 \\
\hline & Female & 34.8 & 28.4 & 7.7 & 23.6 \\
\hline \multirow[t]{2}{*}{$\mathrm{ZAG}$} & Male & 36.7 & 14.7 & 5.4 & 22.1 \\
\hline & Female & 29.5 & 16.4 & 9.7 & 21.7 \\
\hline \multirow[t]{2}{*}{ DEL } & Male & 19.8 & 1.9 & 2.7 & 9.0 \\
\hline & Female & 22.5 & 3.4 & - & 11.3 \\
\hline \multirow[t]{2}{*}{ HON } & Male & 4.1 & - & - & 2.0 \\
\hline & Female & 2.9 & - & - & 1.3 \\
\hline \multirow[t]{2}{*}{ TOK } & Male & 40.7 & 23.3 & 11.1 & 27.2 \\
\hline & Female & 28.4 & 13.5 & 5.1 & 18.4 \\
\hline \multirow[t]{2}{*}{ HAV } & Male & 27.3 & 18.3 & 1.3 & 16.3 \\
\hline & Female & 18.6 & 7.7 & 6.3 & 10.9 \\
\hline \multirow[t]{2}{*}{ OKL } & Male & 29.7 & 17.6 & 11.1 & 24.8 \\
\hline & Female & 34.8 & 20.2 & 10.2 & 28.5 \\
\hline \multirow[t]{2}{*}{ ARI } & Male & 65.6 & 36.6 & 21.4 & 44.8 \\
\hline & Female & 56.3 & 25.4 & 5.7 & 30.5 \\
\hline \multirow[t]{2}{*}{ BUL } & Male & 13.7 & 4.3 & 1.7 & 7.7 \\
\hline & Female & 11.5 & 3.4 & 2.9 & 6.4 \\
\hline \multirow[t]{2}{*}{ Total } & Male & 28.9 & 12.6 & 3.1 & 16.5 \\
\hline & Female & 25.7 & 11.1 & 4.0 & 15.2 \\
\hline
\end{tabular}

Table 18. Overall large-vessel disease prevalence (\%) by sex

\begin{tabular}{lrr}
\hline & Men & Women \\
\hline Component items & & \\
ECG coronary probable & 4.7 & 4.4 \\
ECG coronary possible & 13.1 & 18.4 \\
Possible infarction & 8.3 & 8.3 \\
Angina pectoris & 7.4 & 12.4 \\
Amputation & 2.2 & 1.2 \\
Claudication & 1.6 & 1.4 \\
Stroke & 2.3 & 2.6 \\
Composite items & & \\
Heart vascular disease & 26.2 & 35.0 \\
Leg vascular disease & 3.8 & 2.6 \\
Total LVD & 29.5 & 37.4 \\
Subclass items & & \\
CVD - severe & 6.7 & 5.6 \\
$\quad$ intermediate & 21.7 & 30.7 \\
$\quad$ - stroke only & 1.1 & 1.1 \\
\hline
\end{tabular}

\section{Results}

This section presents the composition of the 14 samples, a comparison of their biometric, clinical and biochemical characteristics and their respective prevalence of large and small vessel disease. Some preliminary results have already been reported $[10,11]$. Relationships, simple and complex, between the explanatory analytical variables and the vascular endpoints are presented for the whole multinational sample and for centres taken individually. Deviations from protocol or missing data in particular instances are noted in the text.

\section{Age, sex and duration composition}

The numbers of men and women with diabetes recruited at each centre are shown in Table 4. The Arizona (Pima Indian) sample was inevitably small because of the limited total number of diabetic patients available in this small population. The distribution by age at examination shown in Table 5 departs from the anticipated one-third in each age sub-segment of the 35-54 band selected, and reflects the difficulties found in many centres in recruiting adequate numbers at the younger end of the scale. As Tables 6 and 7 indicate, this shortfall was chiefly due to limited numbers of younger diabetic patients in the longest-duration ( $\geqslant 14$ years) category; numbers with age at diagnosis under 25 were strikingly low in Hong Kong, Tokyo and Oklahoma samples. To some extent the shortfall is also due to a higher age at interview than age at selection which was due to the time ( $<3$ years) elapsed between sampling and examination.

\section{Biometric, clinical and biochemical characteristics}

The distribution of arterial pressures, the frequency of 'hypertension' (WHO definition) and mean arterial pressures by age appear for each centre in Tables 8, 9 and 10 respectively. About one-third of the participants meet the WHO criteria for hypertension, slightly more in women than men and varying two-fold between centres (Table 9). About half of the hypertensives were taking blood-pressure-lowering therapy at the time of the ascertainment, though the proportion varied enormously among centres between $9 \%$ and almost $90 \%$. Only one-half of this treated group had pressures below $160 / 95$ overall, again with wide variation among centres in 'efficacy' of treatment.

In most centres age-specific mean systolic pressures (Table 10) were higher in women than in men. This difference is least evident in the youngest age group, but increases progressively to the oldest. Mean pressures are low in both sexes in the Tokyo sample and in both American Indian populations.

Mean Body Mass Index (Table 11) ranged between a low of 21.4 in young Tokyo women to 34 in those from the Oklahoma sample. Values were uniformly low in the three Asian centres and indicated marked obesity in the Arizona and Oklahoma participants, approached by the Moscow and Warsaw samples among the European subjects. BMI was greater for women than for men in most age categories, but showed less tendency to increase with age. 
Table 19. Overall large-vessel disease prevalence (\%) by age-group and sex

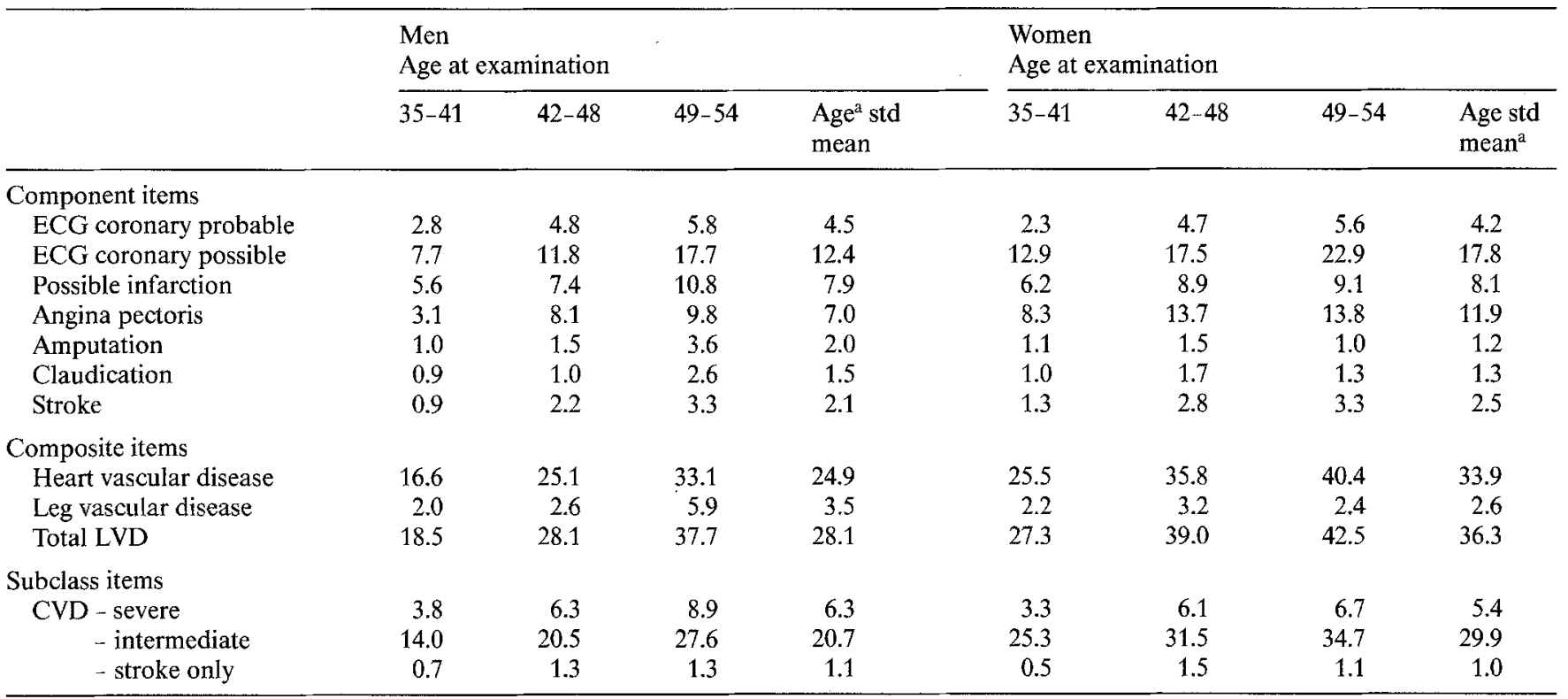

${ }^{a}$ Age-standardized mean is the arithmetic mean of the means for the three age groups

Table 20. Large-vessel disease prevalence (\%) by age group, sex and centre

\begin{tabular}{|c|c|c|c|c|c|}
\hline \multirow[t]{2}{*}{ Centre } & \multirow[t]{2}{*}{ Sex } & \multicolumn{4}{|l|}{ Age group } \\
\hline & & $35-41$ years & $42-48$ years & $49-54$ years & $\begin{array}{l}\text { All } \\
\text { ages }\end{array}$ \\
\hline \multirow[t]{2}{*}{ LON } & Male & 19.7 & 31.6 & 33.6 & 29.1 \\
\hline & Female & 9.4 & 26.4 & 40.8 & 28.8 \\
\hline \multirow[t]{2}{*}{ SWI } & Male & 13.3 & 31.6 & 38.9 & 28.1 \\
\hline & Female & 20.7 & 25.8 & 41.3 & 28.9 \\
\hline \multirow[t]{2}{*}{ BRU } & Male & 8.3 & 11.3 & 30.5 & 19.1 \\
\hline & Female & 13.8 & 26.8 & 36.1 & 26.3 \\
\hline \multirow[t]{2}{*}{ MOS } & Male & 21.2 & 32.1 & 39.6 & 32.7 \\
\hline & Female & 40.0 & 48.1 & 46.7 & 45.4 \\
\hline \multirow[t]{2}{*}{ WAR } & Male & 28.8 & 23.8 & 49.5 & 36.5 \\
\hline & Female & 29.3 & 45.2 & 45.6 & 41.6 \\
\hline \multirow[t]{2}{*}{ BER } & Male & 13.3 & 31.1 & 56.3 & 35.8 \\
\hline & Female & 32.4 & 50.0 & 61.3 & 49.8 \\
\hline \multirow[t]{2}{*}{ ZAG } & Male & 15.4 & 25.0 & 35.3 & 26.1 \\
\hline & Female & 21.1 & 27.1 & 43.1 & 32.2 \\
\hline \multirow[t]{2}{*}{ DEL } & Male & 22.5 & 36.3 & 37.8 & 32.5 \\
\hline & Female & 22.1 & 40.6 & 43.3 & 36.8 \\
\hline \multirow[t]{2}{*}{ HON } & Male & 3.6 & 8.7 & 32.7 & 20.2 \\
\hline & Female & 22.0 & 33.3 & 27.6 & 27.7 \\
\hline \multirow[t]{2}{*}{ TOK } & Male & 8.0 & 17.1 & 15.5 & 14.5 \\
\hline & Female & 23.1 & 28.1 & 18.1 & 21.9 \\
\hline \multirow[t]{2}{*}{$\mathrm{HAV}$} & Male & 32.9 & 39.1 & 42.5 & 38.4 \\
\hline & Female & 47.7 & 61.4 & 49.4 & 52.9 \\
\hline \multirow[t]{2}{*}{ OKL } & Male & 20.0 & 40.4 & 38.7 & 34.8 \\
\hline & Female & 30.2 & 40.3 & 53.6 & 43.3 \\
\hline \multirow[t]{2}{*}{ ARI } & Male & 33.3 & 24.1 & 35.3 & 31.0 \\
\hline & Female & 32.3 & 27.3 & 40.3 & 34.0 \\
\hline \multirow[t]{2}{*}{ BUL } & Male & 17.6 & 26.2 & 38.7 & 30.2 \\
\hline & Female & 23.8 & 46.3 & 41.7 & 38.6 \\
\hline \multirow[t]{2}{*}{ Total } & Male & 18.5 & 28.1 & 37.7 & 29.5 \\
\hline & Female & 27.3 & 39.0 & 42.5 & 37.4 \\
\hline
\end{tabular}
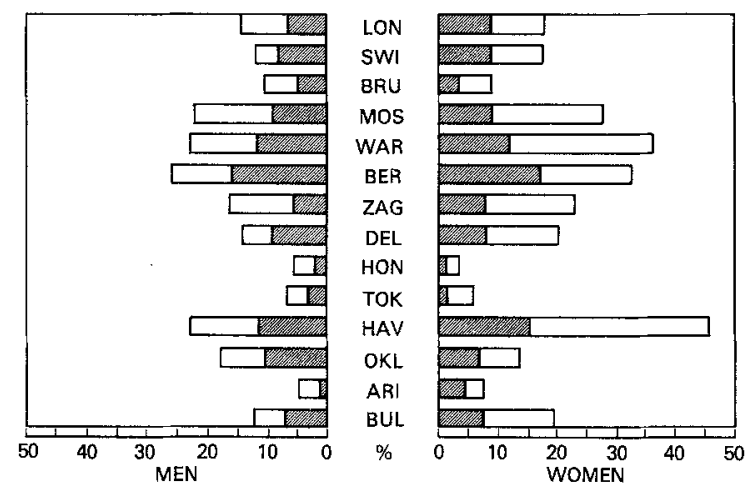

Fig. 1: Prevalence rates for angina pectoris and myocardial infarction from questionnaire data by centre and sex. $\mathbb{Z}$ Possible myocardial infarction; $\square$ angina pectoris
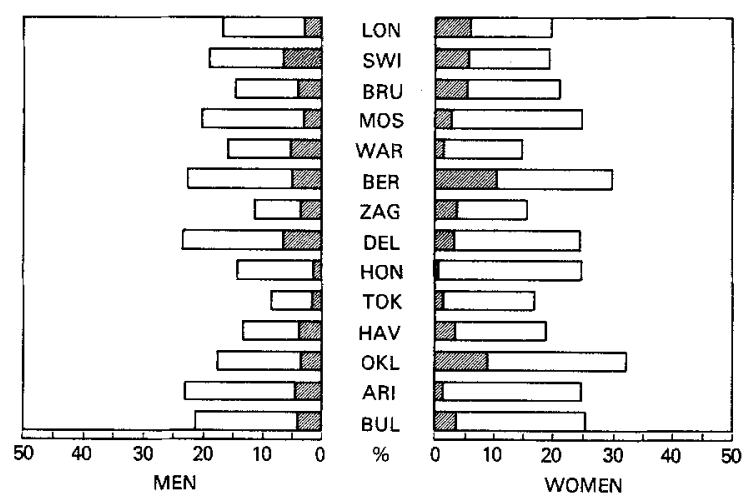

Fig. 2: Prevalence rates for ECG categories 'ECG Coronary Possible' and 'ECG Coronary Probable' by centre and sex. $\mathbb{Z}$ ECG coronary possible; $\square$ ECG coronary probable 
The patterns of cigarette smoking reported from the centres varied widely (Table 12). More men smoked than women. In the three Asian centres most women had never smoked, a pattern shared by Moscow, Bulgaria and Zagreb samples. Two-thirds of the Tokyo men smoked regularly, one-quarter heavily.

Proportionate distribution of plasma cholesterol values by centre, and the age-specific mean total cholesterol levels, (Tables 13 and 14) show relatively low mean values in Arizona, Oklahoma, Tokyo, Bulgaria, Hong Kong and Warsaw samples. Mean values differed little between sexes. Values greater than $7.7 \mathrm{mmol} / 1(300 \mathrm{mg} /$ dl) were found in $8 \%$ overall, occurring with greater frequency in Havana, New Delhi, Moscow, Switzerland and Zagreb samples. In making these comparisons it must be borne in mind that, for logistic reasons, a proportion of centres were unable to obtain estimates from the central laboratories and local estimations were used in these cases (Table 14).

Proportions of patients injecting insulin, taking oral anti-diabetic agents and on neither of these therapies appear by centre and by known duration of diabetes in the successive Tables $15-17$. The proportion receiving
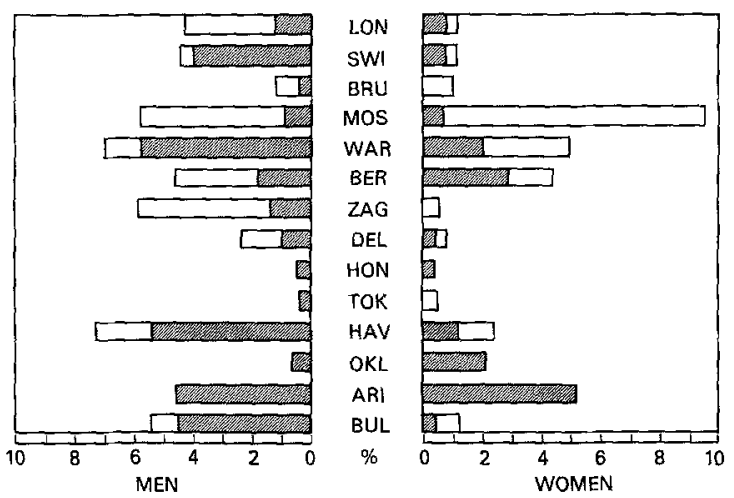

Fig.3: Prevalence rates for Leg Vascular Disease categories, amputation and intermittent claudication by centre and sex. Amputation; $\square$ intermittent claudication insulin increased in successive duration categories. Use of insulin, irrespective of duration, varied from $17.3 \%$ in New Delhi women to $61.3 \%$ for London women. The overall variation was related in some degree to the composition of the groups with respect to age and duration. The 'diet only' group was poorly represented in the long-duration subgroup which contained a relative excess of insulin-injecting patients. In several centres, New Delhi and Oklahoma in particular, relatively few of the long-duration patients were taking insulin injections, an indication of the probable heterogeneity of 'types' of diabetes among samples.

\section{Large-vessel disease}

\section{a) LVD prevalence overall and by centre}

An estimate of the overall prevalence of the large vessel disease endpoints for all centres pooled is presented in Table 18. Rates for individual component, composite and subclass items are indicated. In this table some individuals appear in more than one of the item groupings. Within the CVD composite category, however, the items are mutually exclusive (see Table 2). Men and women are similarly affected, except in the 'Coronary Possible' and 'Angina Pectoris' categories, where the rates for women are about $50 \%$ higher. This difference accounts for the female excess in the prevalence of certain of the composite items in Table 18. Some evidence of LVD was reported for $37.4 \%$ of the women and $29.5 \%$ of the men (total LVD). Rates for claudication, amputation, 'Leg VD' and 'CVD Severe' are low, but slightly higher in men than in women.

These overall rates are presented by age group in Table 19. In men the rates are two to three times higher in the oldest than in the youngest age groups. In women the trend with age is similar, though the gradient is generally less steep than in men; in some categories no gradient is discernible.

Table 21. Overall mean values of systolic blood pressure, body mass index, cholesterol and diabetes duration, by sex, in various categories of LVD

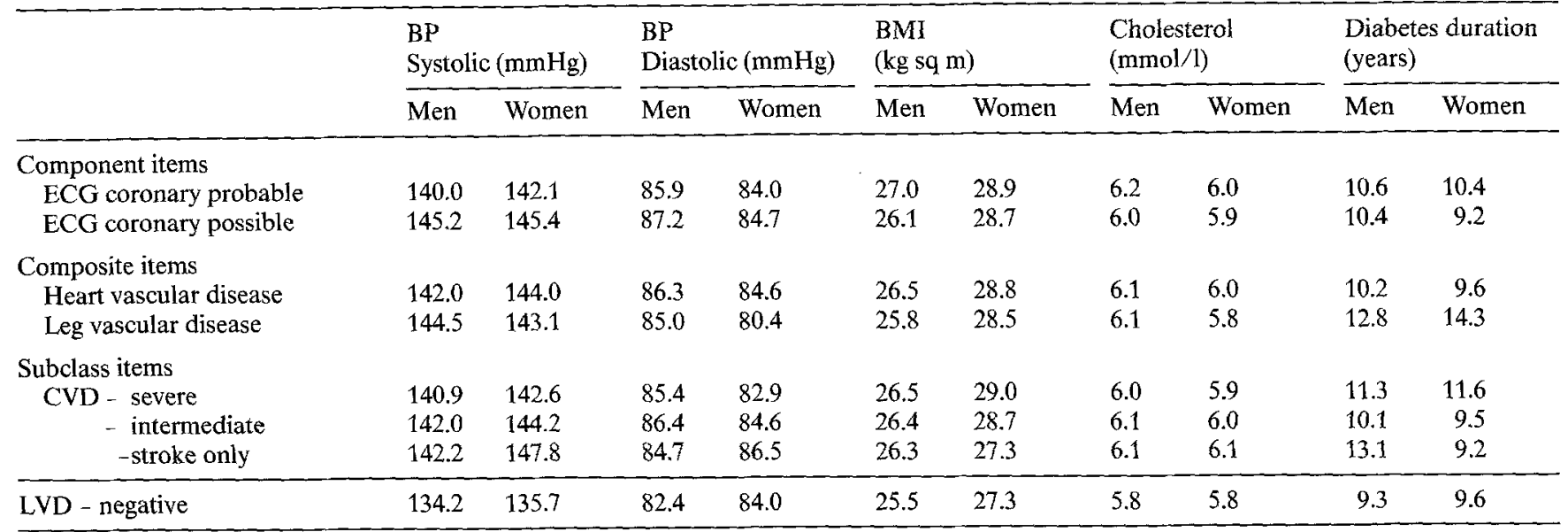


Table 22. Large-vessel disease univariate associations by sex; pooled data

\begin{tabular}{|c|c|c|c|c|c|}
\hline & LVD & Leg VD & $\begin{array}{l}\text { Stroke } \\
\text { only }\end{array}$ & $\begin{array}{l}\text { ECG cor. } \\
\text { probable }\end{array}$ & HVD \\
\hline \multicolumn{6}{|l|}{ Men } \\
\hline Age & $\mathrm{XXX}$ & XXX & $\mathrm{XXX}$ & XXX & $\mathrm{XXX}$ \\
\hline Duration & $\mathrm{XXX}$ & XXX & & XX & XXX \\
\hline Hypertension & XXX & $\mathrm{XX}$ & XXX & XXX & $\mathrm{XXX}$ \\
\hline BMI & $\mathrm{XXX}$ & 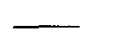 & 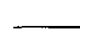 & $\mathrm{XXX}$ & $\mathrm{XXX}$ \\
\hline Smoking & & & - & {[} & - \\
\hline Treatment & & $\mathrm{XXX}$ & & & - \\
\hline Cholesterol & XXX & $\mathrm{XX}$ & XX & $\mathrm{XXX}$ & $\mathrm{XXX}$ \\
\hline \multicolumn{6}{|l|}{ Women } \\
\hline Age & $\mathrm{XXX}$ & XXX & $\mathrm{XXX}$ & $\mathrm{XXX}$ & $\mathrm{XXX}$ \\
\hline Duration & $X X$ & $\mathrm{XXX}$ & & & \\
\hline Hypertension & $\mathrm{XXX}$ & $\mathrm{X}$ & $\mathrm{XXX}$ & $\mathrm{XXX}$ & XXX \\
\hline BMI & $\mathrm{XXX}$ & & $\mathrm{XX}$ & XXX & $\mathrm{XXX}$ \\
\hline Smoking & & & & $\longrightarrow$ & - \\
\hline Treatment & $\bar{x}$ & $\mathrm{XXX}$ & & - & $\overline{Y Y x}$ \\
\hline Cholesterol & XXX & - & $\mathrm{X}$ & XX & XXX \\
\hline
\end{tabular}

Key: $\mathrm{X}=0.01<p<0.05 ; \mathrm{XX}=0.001<p<0.01, \mathrm{XXX}=p<0.001$

Table 23. Standardized multivariate logistic regression coefficients of analytical variables using maximum-likelihood method. Large-vessel disease endpoints; pooled data

\begin{tabular}{|c|c|c|c|c|c|}
\hline & \multicolumn{5}{|c|}{ Dependent variables } \\
\hline & LVD & Leg VD & Stroke & $\begin{array}{l}\text { ECG } \\
\text { coronary } \\
\text { probable }\end{array}$ & HVD \\
\hline \multicolumn{6}{|l|}{ Men } \\
\hline Age & $0.35^{* * *}$ & $0.50 * * *$ & $0.43^{* *}$ & $0.22^{*}$ & $0.31 * * *$ \\
\hline $\begin{array}{l}\text { Duration of } \\
\text { diabetes }\end{array}$ & $0.16^{* * *}$ & $0.22 *$ & 0.08 & $0.22 *$ & $0.12^{*}$ \\
\hline Systolic BP & $0.29 * * *$ & $0.20^{*}$ & $0.36^{* *}$ & 0.13 & $0.29 * * *$ \\
\hline BMI & $0.17^{* * *}$ & 0.01 & 0.03 & $0.29 * * *$ & $0.18^{* * *}$ \\
\hline Smoking & 0.06 & 0.09 & 0.07 & 0.03 & 0.05 \\
\hline Treatment & 0.03 & $0.34 * *$ & 0.03 & -0.07 & 0.004 \\
\hline Cholesterol & $0.18 * * *$ & 0.12 & 0.18 & $0.18^{* *}$ & $0.18 * * *$ \\
\hline \multicolumn{6}{|l|}{ Women } \\
\hline $\begin{array}{l}\text { Age } \\
\text { Duration }\end{array}$ & $0.16^{* * *}$ & -0.04 & 0.22 & $0.24 *$ & $0.16^{* * *}$ \\
\hline of diabetes & 0.08 & $0.42 * * *$ & 0.12 & 0.16 & 0.05 \\
\hline Systolic BP & $0.34 * * *$ & 0.12 & $0.29 * *$ & 0.15 & $0.34 * * *$ \\
\hline BMI & $0.22^{* * *}$ & $0.30^{*}$ & 0.19 & $0.21 * *$ & $0.22^{* * *}$ \\
\hline Smoking & 0.02 & 0.10 & 0.12 & 0.10 & 0.01 \\
\hline Treatment & 0.03 & $0.42^{* *}$ & 0.02 & -0.01 & 0.02 \\
\hline Cholesterol & 0.06 & -0.12 & 0.06 & 0.12 & 0.06 \\
\hline
\end{tabular}

Key: ${ }^{*}=0.01<p<0.05 ;{ }^{* *}=0.001<p<0.01 ;{ }^{* * *}=p<0.001$

The influence of age and sex on 'Total LVD' prevalence in the individual centres is examined in Table 20. With few exceptions rates are higher in women. In some instances this difference is considerable. The rise with age is generally steeper in men.

The category 'Large-Vessel Disease' in Table 20 comprises all the component items. Cultural and linguistic difficulties probably confound comparison of subjective items. Considerable variation was found between centres in the responses to the WHO question- naire for angina, history of myocardial infarction and claudication. Figure 1 displays some of the positive response rates to the arterial disease questionnaire. Tokyo, Hong Kong and Arizona have total rates below $10 \%$, but most other centres cluster around $20 \%$ positive response rates for men and rather more for women. Havana has a conspicuously high rate of $45 \%$ for women, probably arising from confusion between 'chest pain' and 'breast pain' in the Spanish version of the questionnaire.

The more objective Minnesota code rates for ' $\mathrm{ECG}$ Coronary Probable' and 'ECG Coronary Possible' categories, standardized for age, are shown for both sexes (Fig. 2). Abnormalities coded as 'ECG Coronary Probable' varied more than twenty-fold in frequency, ranging from $0.4 \%$ in diabetic women from Hong Kong to $10.5 \%$ in diabetic women from Berlin. Rates are low in both sexes in Hong Kong and Japan, and high in Berlin and Oklahoma women. No major difference exists between the sexes. The ECG-based rates in Figure 2 agree in relative terms with the questionnaire estimates of Fig. 1, but the ratio of 'ECG Coronary Proable' to questionnaire (Possible myocardial infarction) positivity is relatively high for Tokyo and Hong Kong, and low for other centres, particularly Warsaw and Havana. These differences in ratios may represent true variability in the type and frequency of arterial disease manifestations between centres, or may reflect cultural differences in the perception or interpretation of the questionnaires.

Rates for 'ECG Coronary Possible' in women exceed those in men for every centre (Fig. 2). Differences in these 'ECG Coronary Possible' rates should be interpreted with caution, for many non-specific abnormalities of repolarisation contribute to the "Coronary Possible' category, and these abnormalities may vary independently from ischaemic heart disease.

Rates of 'Intermittent Claudication' and 'Amputation' (Fig. 3) are generally low for both sexes; rates for men tend to exceed those for women. 'Claudication' responses were found with high relative frequency in Moscow, Warsaw and Havana. High 'Amputation' rates in males in the latter two centres resembled those in the two American Indian groups, where, anomalously, there is no complaint of claudication. Both are very uncommon in Hong Kong and Tokyo.

\section{b) LVD univariate and multivariate associations}

Table 21 shows the age-standardized mean values of arterial pressure, BMI, total cholesterol and diabetes duration for selected LVD subgroups. Arterial pressures in each LVD subgroup exceed the levels in the 'LVD Negative' group by 6 to $11 \mathrm{mmHg}$ systolic, and by 2 to $5 \mathrm{mmHg}$ diastolic. The mean BMI is slightly higher for 'CVD Positive' than 'Negative' groups, with the exception of 'Stroke Only' groups. Mean cholesterol concentrations in all LVD categories exceed those for the 'LVD Negative' group for both sexes, more so in men than in 
Table 24. Multiple regression analysis: values of $\beta$ coefficients and $\mathrm{F}$ statistics ${ }^{\mathrm{a}}$ by centre and sex. Dependent variable $=$ LVD

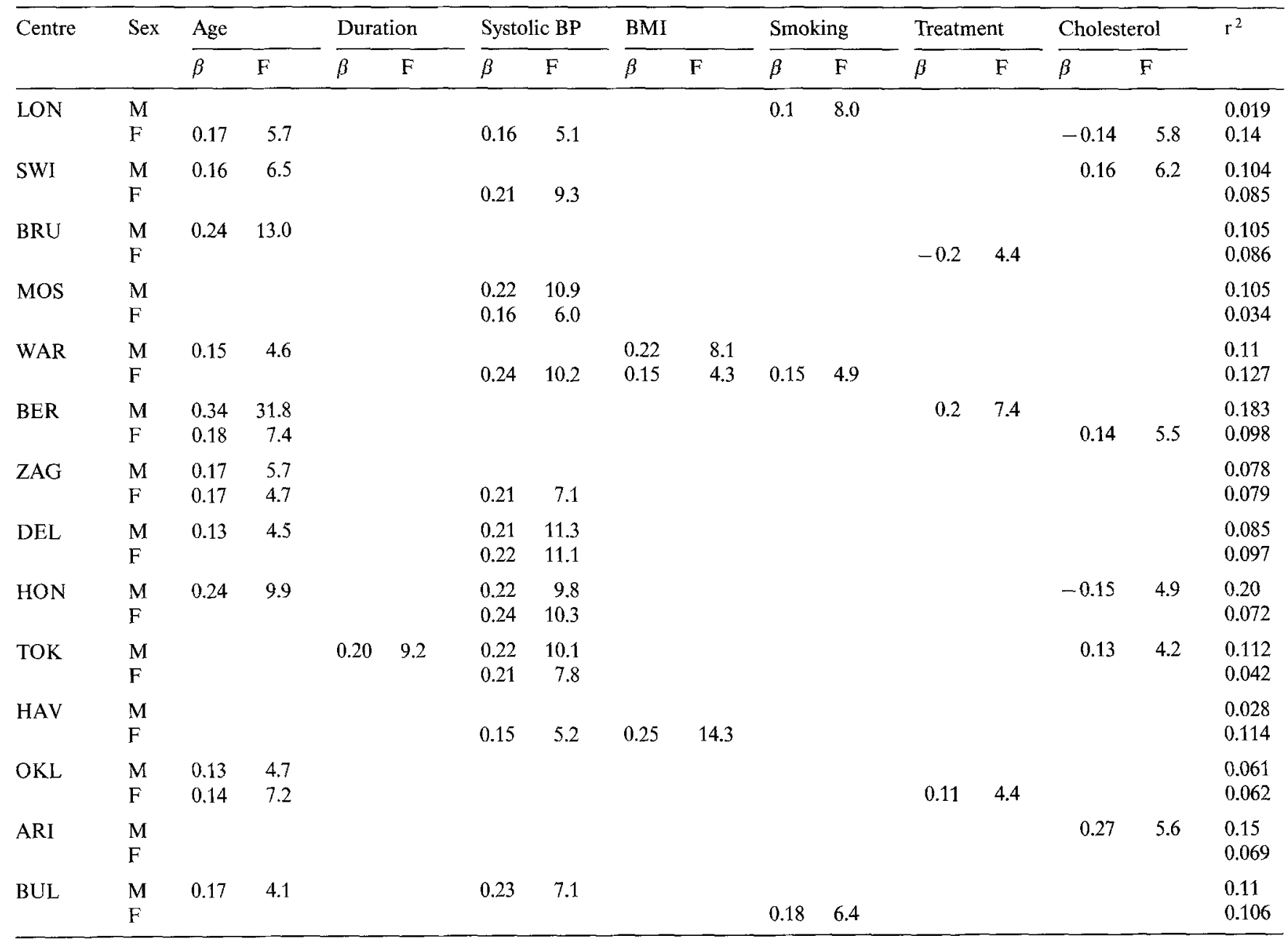

anly values $\geqslant 4$ (where $p \bumpeq 0.05$ or less) are shown

women. Though duration is greater in all LVD subgroups, it is notably different only for 'Leg VD'.

Table 22 summarizes the probability values of correlation coefficients (all correlations are positive), that relate LVD endpoints to a selection of analytical variables. The high levels of statistical significance attached to many of these associations reflect the large numbers of observations and not necessarily the biological importance or strength of the associations. The lack of correlation of the coded smoking class with the various endpoints is particularly striking. Treatment level was coded as insulin, oral agent, or diet-only therapy in declining sequence. The association of treatment with 'Leg VD' therefore indicates that more diabetics so affected were taking insulin or oral agents.

Simple univariate correlations of LVD endpoints with variables such as age, duration and blood pressure are difficult to interpret since the latter variables are often significantly correlated with each other. Furthermore, trends in the group as a whole may arise from differences between centres. For example, the Tokyo sample has both low BMI values and low rates of LVD, while the Oklahoma sample shows the reverse. This might confer upon the group as a whole a strong association between BMI and LVD which does not necessarily exist within individual centres. The independent contribution of each to LVD can also be evaluated by logistic regression analysis. This statistical technique adjusts for the correlation of independent variables, and then examines the association with a dependent variable that has only two possible values (absent or present). The strength of such adjusted relationships is indicated by the values of the $\beta$-coefficients for the independent variables.

Various LVD endpoints were examined in turn as dependent variables and related to age, duration of diabetes, systolic blood pressure, BMI, current smoking habit, treatment regimen and plasma cholesterol concentration in a forward stepwise logistic regression analysis. The values of the standardized $\beta$ coefficients with their significance levels are shown in Table 23. Among the diabetic men, age, duration of diabetes, systolic blood pressure, BMI and cholesterol level are independently associated with 'Total LVD'. Similar asso- 
Table 25. Multiple regression analysis: values of beta coefficients and $F$ statistics ${ }^{\mathrm{a}}$ by centre and sex. Dependent variable $=\mathrm{HVD}$

\begin{tabular}{|c|c|c|c|c|c|c|c|c|c|c|c|c|c|c|c|c|}
\hline \multirow[t]{2}{*}{ Centre } & \multirow[t]{2}{*}{ Sex } & \multicolumn{2}{|l|}{ Age } & \multicolumn{2}{|c|}{ Duration } & \multicolumn{2}{|c|}{ Systolic BP } & \multicolumn{2}{|l|}{ BMI } & \multicolumn{2}{|c|}{ Smoking } & \multicolumn{2}{|c|}{ Treatment } & \multicolumn{2}{|c|}{ Cholesterol } & \multirow[t]{2}{*}{$r$} \\
\hline & & $\beta$ & $\mathrm{F}$ & $\beta$ & $F$ & $\beta$ & $F$ & $\beta$ & $F$ & $\beta$ & $\mathrm{F}$ & $\beta$ & $\mathrm{F}$ & $\beta$ & $\mathrm{F}$ & \\
\hline LON & $\begin{array}{l}\mathrm{M} \\
\mathrm{F}\end{array}$ & & & & & 0.17 & 5.4 & & & & & & & -0.16 & 6.0 & $\begin{array}{l}0.018 \\
0.149\end{array}$ \\
\hline SWI & $\begin{array}{l}\mathrm{M} \\
\mathrm{F}\end{array}$ & 0.13 & 4.3 & & & 0.22 & 9.8 & & & & & & & 0.15 & 5.3 & $\begin{array}{l}0.068 \\
0.092\end{array}$ \\
\hline BRU & $\begin{array}{l}\mathrm{M} \\
\mathrm{F}\end{array}$ & 0.25 & 13.9 & 0.18 & 4.4 & & & & & & & -0.21 & 4.9 & & & $\begin{array}{l}0.094 \\
0.092\end{array}$ \\
\hline MOS & $\begin{array}{l}\mathrm{M} \\
\mathrm{F}\end{array}$ & & & & & $\begin{array}{l}0.21 \\
0.15\end{array}$ & $\begin{array}{r}10.0 \\
5.5\end{array}$ & & & & & & & & & $\begin{array}{l}0.095 \\
0.040\end{array}$ \\
\hline WAR & $\begin{array}{l}\mathrm{M} \\
\mathrm{F}\end{array}$ & & & & & $\begin{array}{l}0.18 \\
0.24\end{array}$ & $\begin{array}{r}6.1 \\
10.7\end{array}$ & 0.15 & 4.4 & & & & & & & $\begin{array}{l}0.110 \\
0.117\end{array}$ \\
\hline BER & $\begin{array}{l}\mathrm{M} \\
\mathrm{F}\end{array}$ & $\begin{array}{l}0.33 \\
0.16\end{array}$ & $\begin{array}{r}30.1 \\
6.1\end{array}$ & & & 0.17 & 6.1 & & & & & & & & & $\begin{array}{l}0.116 \\
0.102\end{array}$ \\
\hline ZAG & $\begin{array}{l}M \\
F\end{array}$ & 0.19 & 7.5 & & & 0.22 & 8.5 & & & & & & & & & $\begin{array}{l}0.093 \\
0.079\end{array}$ \\
\hline DEL & $\begin{array}{l}M \\
F\end{array}$ & & & & & $\begin{array}{l}0.22 \\
0.22\end{array}$ & $\begin{array}{l}12.0 \\
11.1\end{array}$ & & & & & & & & & $\begin{array}{l}0.082 \\
0.099\end{array}$ \\
\hline HON & $\begin{array}{l}\mathrm{M} \\
\mathrm{F}\end{array}$ & 0.22 & 8.4 & & & $\begin{array}{l}0.24 \\
0.24\end{array}$ & $\begin{array}{l}11.3 \\
10.2\end{array}$ & & & & & & & & & $\begin{array}{l}0.216 \\
0.077\end{array}$ \\
\hline TOK & $\begin{array}{l}\mathrm{M} \\
\mathrm{F}\end{array}$ & & & 0.18 & 6.2 & $\begin{array}{l}0.21 \\
0.20\end{array}$ & $\begin{array}{l}9.6 \\
6.6\end{array}$ & & & & & & & 0.14 & 4.7 & $\begin{array}{l}0.115 \\
0.036\end{array}$ \\
\hline HAV & $\begin{array}{l}\mathrm{M} \\
\mathrm{F}\end{array}$ & & & & & & & 0.26 & 15.6 & & & $\begin{array}{r}-0.16 \\
0.14\end{array}$ & $\begin{array}{l}5.2 \\
4.6\end{array}$ & & & $\begin{array}{l}0.046 \\
0.114\end{array}$ \\
\hline OKL & $\begin{array}{l}M \\
F\end{array}$ & $\begin{array}{l}0.14 \\
0.17\end{array}$ & $\begin{array}{l}5.1 \\
9.7\end{array}$ & & & & & & & & & & & & & $\begin{array}{l}0.052 \\
0.043\end{array}$ \\
\hline ARI & $\begin{array}{l}\mathrm{M} \\
\mathrm{F}\end{array}$ & & & & & 0.24 & 4.3 & & & & & & & 0.26 & 5.1 & $\begin{array}{l}0.147 \\
0.061\end{array}$ \\
\hline BUL & $\begin{array}{l}M \\
F\end{array}$ & & & & & $\begin{array}{l}0.23 \\
0.20\end{array}$ & $\begin{array}{l}7.2 \\
6.1\end{array}$ & & & & & & & & & $\begin{array}{l}0.107 \\
0.062\end{array}$ \\
\hline
\end{tabular}

${ }^{a}$ Only values $\geqslant 4.0$ (where $p \bumpeq 0.05$ or less) are shown

ciations were also seen for HVD. For women, only age, systolic blood pressure and BMI are independently associated with 'Total LVD'. Age is quantitatively a less important determinant for the women, and hypertension contributes relatively more to the associations. The apparent lack of an association between smoking and LVD must be interpreted with caution. Rates of current smoking may be lowered because those known to have LVD may have altered their habits. However, further analysis did not reveal an excess of former smokers among those with LVD.

Tables 24 and 25 present the results of multiple regression analysis, with dependent variables LVD and HVD respectively, within centres. For simplicity, only 'significant' independent associations are shown (i.e. where the F statistic was 4.0 or more). In both cases, the most consistent association is with systolic blood pressure, followed by age and, much less frequently, by plasma cholesterol. Nevertheless, even for blood pressure, there are some centres where significance was not achieved for either sex and others where it was achieved for only one sex. Duration, BMI and smoking were only rarely significant variables.

\section{Small-vessel disease}

a) SVD prevalence overall and by centre

The overall prevalence of retinal and renal SVD endpoints by sex and diabetes duration is shown in Table 26 . The frequency of the most severe ocular abnormality, 'Retinopathic Blindness', rises from about $1 \%$ for less than 7 years duration to about $6 \%$ in patients with more than 14 years duration. A similar sharp rise in the prevalence rate occurs for the 'New Vessels' category. For the various categories of 'Red Lesions' and both types of exudate there is, however, a more even rise with increasing duration. 'Hard Exudates' were recorded three to four times as frequently as 'Soft Exudates'. The composite items of small vessel disease of the eye (SVDE) appear at the bottom of Table 26. Rates are similar in the two sexes. The prevalence of 'Severe SVDE' and 'Medium SVDE' categories increases with diabetes duration, but 'Minimal SVDE' prevalence remains near $6 \%$ for all durations.

Combining the classes of mild and severe disease of the kidney (SVDK), about $20 \%$ of diabetic patients in 
the shortest duration category were recorded as having proteinuria, two thirds of them with the lesser grade (Table 26). The frequency of the lesser grade of proteinuria changes little with increasing duration, unlike that

Table 26. Overall small-vessel disease prevalence (\%) by duration and sex

\begin{tabular}{|c|c|c|c|c|}
\hline & & \multicolumn{3}{|c|}{$\begin{array}{l}\text { Duration of diabetes mellitus } \\
\text { (years) }\end{array}$} \\
\hline & & $0-6$ & $7-13$ & $14+$ \\
\hline \multicolumn{5}{|l|}{ Component items } \\
\hline \multirow[t]{2}{*}{ Retinopathic blindness } & Male & 0.9 & 1.1 & 6.5 \\
\hline & Female & 0.4 & 1.3 & 5.9 \\
\hline \multirow[t]{2}{*}{ New vessels } & Male & 1.2 & 1.9 & 8.0 \\
\hline & Female & 0.5 & 1.4 & 8.3 \\
\hline \multirow[t]{2}{*}{ Large red lesions } & Male & 0.7 & 2.9 & 8.6 \\
\hline & Female & 0.4 & 2.1 & 6.5 \\
\hline \multirow[t]{2}{*}{ Medium red lesions } & Male & 6.2 & 14.6 & 31.6 \\
\hline & Female & 4.3 & 12.9 & 28.7 \\
\hline \multirow[t]{2}{*}{ Small red lesions } & Male & 9.0 & 21.7 & 46.4 \\
\hline & Female & 6.5 & 22.4 & 42.0 \\
\hline \multirow[t]{2}{*}{ Hard exudates } & Male & 5.9 & 13.7 & 30.0 \\
\hline & Female & 4.1 & 13.3 & 27.2 \\
\hline \multirow[t]{2}{*}{ Soft exudates } & Male & 1.7 & 4.5 & 8.6 \\
\hline & Female & 1.2 & 3.6 & 6.8 \\
\hline \multicolumn{5}{|l|}{ Composite items } \\
\hline \multirow[t]{2}{*}{ Severe SVD of kidney } & Male & 5.8 & 8.6 & 17.7 \\
\hline & Female & 4.5 & 7.8 & 14.8 \\
\hline \multirow[t]{2}{*}{ Mild SVD of kidney } & Male & 11.3 & 14.0 & 15.4 \\
\hline & Female & 15.1 & 17.9 & 16.8 \\
\hline \multirow[t]{2}{*}{ Severe SVD of eye } & Male & 2.2 & 4.8 & 16.6 \\
\hline & Female & 1.1 & 4.1 & 14.7 \\
\hline \multirow[t]{2}{*}{ Medium SVD of eye } & Male & 11.6 & 24.4 & 45.4 \\
\hline & Female & 8.9 & 25.8 & 41.4 \\
\hline \multirow[t]{2}{*}{ Minimal SVD of eye } & Male & 4.0 & 6.5 & 6.0 \\
\hline & Female & 4.2 & 6.3 & 6.8 \\
\hline
\end{tabular}

of the heavier grade, which is three times as common with longer duration. The role of duration appears to be less striking for total SVDK than for SVDE. This finding is largely due to the lesser degrees of positivity for SVDK, and may represent falsely positive or non-specific causes of proteinuria in some of the populations examined. 'Severe SVDK' also occurs a little more frequently in men, a relationship which reverses for 'Mild SVDK'.

Mean values for the systolic blood pressure, cholesterol and body mass index for the various SVD categories by duration group appear in Table 27. Mean systolic blood pressures are higher in the more severe categories of SVD eye and kidney endpoints, especially in the longest duration group. The mean diastolic blood pressure trends (not shown) are similar for SVDK, but less evident for SVDE. Mean cholesterol levels are higher in patients with the severe grades of SVDE, but only in the longest duration groups and more evidently in women. A similar relationship between mean cholesterol and severity, as well as duration, is shown in respect to SVDK. In neither sex does mean BMI show a relationship with the presence or severity of SVDE or SVDK, though for retinal disease BMI tends to be slightly higher in unaffected subjects.

The prevalence rates of 'Retinopathic Blindness' and 'Soft Exudates' by centre are shown in Table 28. Rates for retinopathic blindness rise substantially where duration exceeds 14 years. The reported rates are similar in most centres, the exception being Arizona women (low), Bulgarian men (high) and the early appearance of blindness in the Tokyo sample. Differences in reported prevalence of 'Soft Exudates' could well arise from observer variation. In the longest duration group, for example, 'Soft Exudates' apparently range in prevalence from zero to $26 \%$.

Table 27. Overall mean levels of systolic blood pressure, cholesterol and body mass index by duration of diabetes and sex for small vessel disease composite items

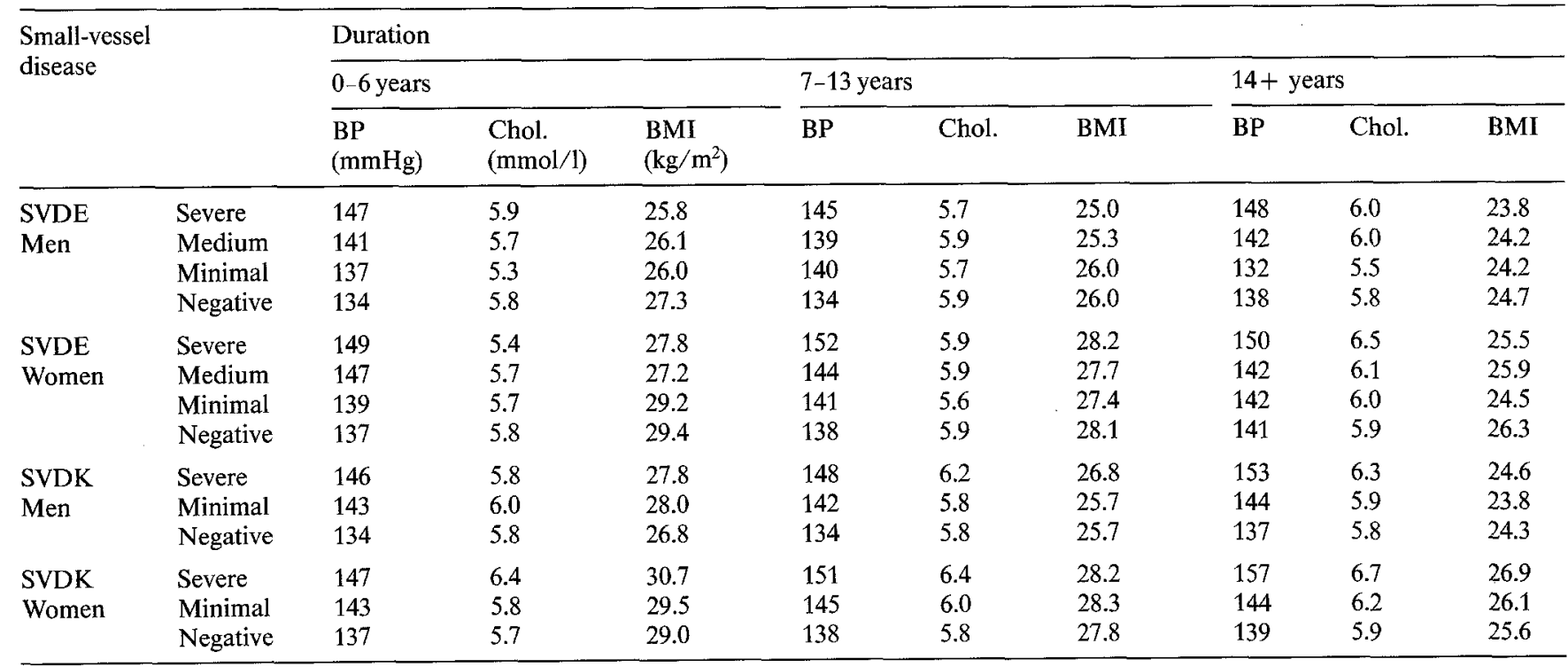


Table 28. Prevalence (\%) of retinopathic blindness and soft exudates: by duration, sex and centre

\begin{tabular}{|c|c|c|c|c|c|c|c|}
\hline \multirow[t]{3}{*}{ Centre } & \multirow[t]{3}{*}{ Sex } & \multicolumn{6}{|c|}{ Duration of diabetes } \\
\hline & & \multicolumn{2}{|c|}{$0-6$ years } & \multicolumn{2}{|c|}{$7-13$ years } & \multicolumn{2}{|c|}{$14+$ years } \\
\hline & & $\mathrm{RP}^{\mathrm{a}}$ & $S E^{b}$ & $\mathrm{RP}$ & $\mathrm{SE}$ & $\mathrm{RP}$ & SE \\
\hline \multirow[t]{2}{*}{ LON } & Male & - & - & 1.2 & - & 3.1 & 3.1 \\
\hline & Female & 1.4 & 1.4 & - & 1.3 & 5.4 & 1.1 \\
\hline \multirow[t]{2}{*}{ SWI } & Male & - & 1.0 & 1.1 & 2.2 & 11.8 & 5.4 \\
\hline & Female & - & 1.1 & - & 1.3 & 8.7 & 7.6 \\
\hline \multirow[t]{2}{*}{ BRU } & Male & 1.7 & 0.9 & - & 3.8 & 4.4 & 2.9 \\
\hline & Female & - & - & - & - & 8.5 & 3.4 \\
\hline \multirow[t]{2}{*}{ MOS } & Male & - & - & - & - & 5.2 & 5.2 \\
\hline & Female & - & - & 0.9 & - & 5.2 & 1.7 \\
\hline \multirow[t]{2}{*}{ WAR } & Male & 1.3 & 1.3 & 1.3 & 1.3 & 2.2 & 2.2 \\
\hline & Female & - & - & - & - & 6.9 & 3.0 \\
\hline \multirow[t]{2}{*}{ BER } & Male & - & - & - & 3.1 & 3.0 & 8.9 \\
\hline & Female & - & - & - & 1.1 & 3.3 & 5.5 \\
\hline \multirow[t]{2}{*}{$\mathrm{ZAG}$} & Male & 1.0 & - & - & 4.4 & 5.4 & 14.3 \\
\hline & Female & - & 1.1 & - & 11.5 & 9.7 & 3.2 \\
\hline \multirow[t]{2}{*}{ DEL } & Male & - & 4.5 & 1.9 & 7.7 & 4.1 & 23.0 \\
\hline & Female & 0.8 & 3.3 & - & 6.9 & 3.4 & 8.5 \\
\hline \multirow[t]{2}{*}{$\mathrm{HON}$} & Male & 1.0 & 1.0 & - & - & 7.0 & - \\
\hline & Female & 1.0 & - & 3.6 & - & 7.9 & 5.3 \\
\hline \multirow[t]{2}{*}{ TOK } & Male & 4.4 & 3.3 & 2.2 & 10.0 & 7.4 & 20.4 \\
\hline & Female & 1.1 & 2.3 & 5.4 & 5.4 & 5.1 & 15.4 \\
\hline \multirow[t]{2}{*}{ HAV } & Male & - & 1.1 & 2.2 & 4.3 & 7.8 & 2.6 \\
\hline & Female & - & - & - & 2.2 & 7.5 & 3.8 \\
\hline \multirow[t]{2}{*}{ OKL } & Male & 1.1 & 0.6 & - & - & 7.4 & - \\
\hline & Female & - & - & 1.2 & - & 8.2 & - \\
\hline \multirow[t]{2}{*}{ ARI } & Male & - & 6.3 & 2.4 & 12.2 & 7.1 & 14.3 \\
\hline & Female & - & - & 4.2 & 5.6 & - & 20.0 \\
\hline \multirow[t]{2}{*}{ BUL } & Male & 1.1 & 6.3 & 2.9 & 17.4 & 20.7 & 24.1 \\
\hline & Female & 1.0 & 7.3 & 2.3 & 16.1 & 2.9 & 26.5 \\
\hline
\end{tabular}

${ }^{\mathrm{a}} \mathrm{RP}=$ retinopathic blindness; ${ }^{\mathrm{b}} \mathrm{SE}=$ soft exudates

Table 29 presents the different severities of composite SVDE by centre and sex. Within each centre, the rates increase with duration and there are no systematic sex differences.

Centre-by-centre prevalence of SVDK is shown in Table 30 for the severe and mild categories. The rates of 'Severe SVDK' are similar in magnitude to those for 'Severe SVDE'. The variation between individual centres for SVDK is greater than that for SVDE. 'Severe SVDK' in long duration diabetes, for example, varies from a low of $2.4 \%$ in Hong Kong to high values of $37.0 \%$ in Oklahoma and 34\% in Tokyo men, respectively.

\section{b) SVD univariate and multivariate associations}

The univariate probability values for correlation of small-vessel disease with selected analytical variables appear in Table 31. Duration, systolic blood pressure or hypertension, and treatment modality are highly correlated with the SVDE and SVDK endpoints, though only the correlation coefficient for duration/SVDE exceeds 0.4 and most correlations are of low order. A negative relationship is found between BMI and SVDE, but not between BMI and SVDK. Diastolic blood pressure and cholesterol values are positively correlated with SVDK, but not with SVDE. Finally, current cigarette smoking is not associated with small-vessel disease of the eye or kidney in women, but is associated with SVDK in men, though with a low degree of significance $(0.01<p$ $<0.05$ ).

Multiple linear regression analysis of the relationship between the analytical variables and SVDE and SVDK endpoints (Table 32) shows the influence of diabetes duration on both, but more particularly upon SVDE. The associations with systolic blood pressure are stronger for SVDK that for SVDE. The negative relationship found for BMI in the univariate analysis survives multiple regression analysis. The magnitudes of the $\beta$-coefficients suggest that duration, followed by systolic blood pressure and treatment, are the most important factors in SVDE, and that the analytical model is able to predict more than $20 \%$ of the variance. For SVDK, duration, systolic blood pressure and cholesterol retain strong independent associations for both sexes, but only $8 \%$ or less of the variance is explained in this model.

Tables 33 and 34 present the results of multiple regression analysis, with dependent variables SVDE and SVDK respectively, within centres. As in Tables 23 and 24, only 'significant' independent associations are shown.

For SVDE all centres and both sexes, with the exception of men in Hong Kong and Havana, show significant associations with duration. There are significant associations in over $50 \%$ of centre/sex groups with systolic blood pressure and treatment category. Associations with BMI occur in only a few centres and in those are uniformly inverse. There are few significant correlations with age, smoking and cholesterol.

For SVDK the most consistent correlation is with systolic blood pressure, Zagreb being the only centre where no significant correlation occurred in either sex. In 12 of the 28 centre/sex groups there were significant correlations with duration and/or cholesterol. As with the pooled data, the proportion of the variance explained by the independent variables within centres was generally lower for SVDK than for SVDE.

\section{Discussion}

Arterial disease affecting vessels of large and small calibre is a characteristic feature of human diabetes. Vascular disease of the retina and of the renal glomerulus are hallmarks of diabetes mellitus. On the other hand, macrovascular disease is qualitatively similar in diabetic and non-diabetic persons, although in Western societies it affects diabetics earlier and more extensively than non-diabetics. Abundant evidence from mortality studies, autopsy data and clinical morbidity compari- 
Table 29. Grades of small-vessel disease of eye (SVDE): prevalence (\%) by duration, sex and centre

\begin{tabular}{|c|c|c|c|c|c|c|c|c|c|c|}
\hline \multirow[t]{3}{*}{ Centre } & \multirow[t]{3}{*}{ Sex } & \multicolumn{9}{|c|}{ Duration of diabetes } \\
\hline & & \multicolumn{3}{|c|}{$0-6$ years } & \multicolumn{3}{|c|}{$7-13$ years } & \multicolumn{3}{|c|}{$14+$ years } \\
\hline & & $\begin{array}{l}\text { Severe } \\
\text { SVDE }\end{array}$ & $\begin{array}{l}\text { Medium } \\
\text { SVDE }\end{array}$ & $\begin{array}{l}\text { Minimum } \\
\text { SVDE }\end{array}$ & $\begin{array}{l}\text { Severe } \\
\text { SVDE }\end{array}$ & $\begin{array}{l}\text { Medium } \\
\text { SVDE }\end{array}$ & $\begin{array}{l}\text { Minimum } \\
\text { SVDE }\end{array}$ & $\begin{array}{l}\text { Severe } \\
\text { SVDE }\end{array}$ & $\begin{array}{l}\text { Medium } \\
\text { SVDE }\end{array}$ & $\begin{array}{l}\text { Minimum } \\
\text { SVDE }\end{array}$ \\
\hline \multirow[t]{2}{*}{ LON } & Male & 2.7 & 16.0 & 2.7 & 4.9 & 19.8 & 9.9 & 13.5 & 47.9 & 5.2 \\
\hline & Female & 4.3 & 10.1 & 4.3 & - & 29.1 & 11.4 & 14.4 & 40.0 & 15.6 \\
\hline \multirow[t]{2}{*}{ SWI } & Male & 1.0 & 8.3 & 5.2 & 1.1 & 14.8 & 5.7 & 27.8 & 38.9 & 4.4 \\
\hline & Female & - & 6.9 & 3.4 & 1.3 & 19.5 & 3.9 & 21.1 & 37.8 & 10.0 \\
\hline \multirow[t]{2}{*}{ BRU } & Male & 3.5 & 2.6 & 4.3 & 1.9 & 15.4 & 3.8 & 11.8 & 45.6 & 16.2 \\
\hline & Female & - & 3.6 & 1.2 & - & 29.3 & 12.2 & 19.0 & 31.0 & 19.0 \\
\hline \multirow{2}{*}{ MOS } & Male & 1.3 & 23.7 & 9.2 & 3.3 & 28.3 & 6.5 & 12.1 & 51.7 & 6.9 \\
\hline & Female & - & 7.0 & 12.0 & 0.9 & 26.1 & 9.6 & 13.8 & 41.4 & 8.6 \\
\hline \multirow[t]{2}{*}{ WAR } & Male & 2.7 & 9.5 & 1.4 & 1.3 & 26.3 & 14.5 & 5.7 & 55.7 & 8.0 \\
\hline & Female & 1.4 & 4.2 & 5.6 & 1.4 & 19.7 & 12.7 & 8.3 & 49.0 & 5.2 \\
\hline \multirow[t]{2}{*}{ BER } & Male & - & 3.4 & 1.1 & 4.3 & 15.2 & 1.1 & 18.6 & 51.5 & 3.1 \\
\hline & Female & - & 2.4 & - & 3.3 & 20.7 & 1.1 & 11.4 & 54.5 & 1.1 \\
\hline \multirow[t]{2}{*}{ ZAG } & Male & 5.2 & 8.3 & 5.2 & 6.0 & 37.3 & 9.0 & 22.6 & 54.7 & - \\
\hline & Female & 2.3 & 14.8 & 5.7 & 8.2 & 32.8 & 3.3 & 25.8 & 35.5 & 3.2 \\
\hline \multirow{2}{*}{ DEL } & Male & 0.9 & 9.9 & 0.9 & 3.9 & 16.7 & 2.0 & 14.1 & 31.0 & 2.8 \\
\hline & Female & 0.8 & 3.4 & 2.5 & 3.5 & 9.4 & 3.5 & 6.8 & 20.3 & 1.7 \\
\hline \multirow[t]{2}{*}{$\mathrm{HON}$} & Male & 1.0 & 18.4 & 7.1 & 1.8 & 21.4 & 10.7 & 9.8 & 29.3 & 7.3 \\
\hline & Female & 1.9 & 15.5 & 3.9 & 3.7 & 23.2 & 4.9 & 13.9 & 41.7 & 8.3 \\
\hline \multirow[t]{2}{*}{ TOK } & Male & 7.7 & 14.3 & 9.9 & 13.5 & 20.2 & 10.1 & 17.0 & 47.2 & 9.4 \\
\hline & Female & 2.3 & 18.2 & 8.0 & 9.6 & 27.4 & 16.4 & 23.7 & 31.6 & 5.3 \\
\hline \multirow[t]{2}{*}{ HAV } & Male & - & 18.4 & - & 3.2 & 34.4 & 1.1 & 10.5 & 42.1 & 3.9 \\
\hline & Female & - & 14.0 & 1.2 & - & 27.5 & - & 8.9 & 39.2 & 1.3 \\
\hline \multirow[t]{2}{*}{ OKL } & Male & 1.7 & 9.1 & 1.7 & 4.4 & 23.5 & 11.8 & 18.5 & 40.7 & 7.4 \\
\hline & Female & - & 5.2 & 3.2 & 12.2 & 19.5 & 4.9 & 16.7 & 43.8 & 2.1 \\
\hline \multirow[t]{2}{*}{ ARI } & Male & - & 20.0 & 3.3 & 9.8 & 29.3 & 4.9 & 35.7 & 21.4 & 7.1 \\
\hline & Female & 4.2 & 6.3 & 8.3 & 8.5 & 28.2 & 5.6 & 18.2 & 42.4 & 15.2 \\
\hline \multirow[t]{2}{*}{ BUL } & Male & 2.1 & 12.6 & 5.3 & 8.7 & 44.9 & 2.9 & 32.8 & 50.0 & 5.2 \\
\hline & Female & 2.1 & 17.7 & 3.1 & 5.7 & 51.7 & 3.4 & 18.2 & 56.1 & - \\
\hline
\end{tabular}

sons show this excess in diabetic subjects [1]. It may also be present in non-Western societies, including industrialized countries such as Japan and developing regions such as Africa [12-16]. However, in these other areas, absolute rates of atherosclerotic arterial disease, particularly coronary heart disease in diabetic subjects, have been reported at much lower rates than in corresponding North American or European diabetic populations. Indeed, the differential susceptibility of diabetes resembles the striking regional, national or ethnic variation in arterial disease prevalence among the respective general populations $[2,16]$.

Prospective heart disease studies that included diabetic persons have until recently been restricted largely to Western population groups. The Tecumseh and Framingham communities in the USA, civil servants in the Whitehall Study in Britain and similar populations in Israel have all reported increased prevalence and incidence of cardiac disease among their diabetic compared with their non-diabetic subjects [17-20]. In some populations, differences in large-vascular disease rates are especially great for diabetic women $[1,21]$. Factors among the population at large that are positively associated with increased risk of CVD also appear to oper- ate within diabetic populations. They include elevated total serum cholesterol concentrations (or alterations in the levels of the low-density lipoprotein or high-density lipoprotein constituent fractions), elevated arterial blood pressure and cigarette smoking [15]. The number of diabetic patients included in these large-scale population studies has been relatively small, but support the view that increased atherosclerotic risk in the diabetic cannot be explained simply by an increased prevalence of known risk factors [1].

Many reports of prevalence and severity of vascular disease in diabetic patients have emanated from special clinical groups, usually hospital-based. The potential for bias is high in such studies. Special diabetes clinics often attract the cases with more complications, those in a particular section of society, or diabetics with particular manifestations of the disease, reflecting the interests or expertise of those offering clinical care. In many such clinical series reports, the more frequently attending patient (perhaps the most complicated or the most careful) is inevitably more likely to be included than the less frequent attender. Unless special measures are taken, such groups of cases (and controls) may misrepresent the study populations [22]. 
Table 30. Grades of small vessel disease of the kidney: prevalence (\%) by duration, sex and centre

\begin{tabular}{|c|c|c|c|c|c|c|c|}
\hline \multirow[t]{3}{*}{ Centre } & \multirow[t]{3}{*}{ Sex } & \multicolumn{6}{|c|}{ Duration of diabetes } \\
\hline & & \multicolumn{2}{|c|}{$0-6$ years } & \multicolumn{2}{|c|}{$7-13$ years } & \multicolumn{2}{|c|}{$14+$ years } \\
\hline & & $\begin{array}{l}\text { Severe } \\
\text { SVDK }\end{array}$ & $\begin{array}{l}\text { Mild } \\
\text { SVDK }\end{array}$ & $\begin{array}{l}\text { Severe } \\
\text { SVDK }\end{array}$ & $\begin{array}{l}\text { Mild } \\
\text { SVDK }\end{array}$ & $\begin{array}{l}\text { Severe } \\
\text { SVDK }\end{array}$ & $\begin{array}{l}\text { Mild } \\
\text { SVDK }\end{array}$ \\
\hline \multirow[t]{2}{*}{ LON } & Male & 2.7 & 10.8 & 3.7 & 17.1 & 4.2 & 16.7 \\
\hline & Female & 2.9 & 11.4 & 3.8 & 16.3 & 6.5 & 19.4 \\
\hline \multirow[t]{2}{*}{ SWI } & Male & 7.3 & 22.9 & 8.0 & 17.2 & 21.5 & 11.8 \\
\hline & Female & 4.7 & 23.3 & 9.1 & 28.6 & 20.7 & 16.3 \\
\hline \multirow[t]{2}{*}{ BRU } & Male & - & 2.6 & - & 5.8 & 10.3 & 7.4 \\
\hline & Female & - & 3.6 & 2.5 & 2.5 & 6.8 & 5.1 \\
\hline \multirow[t]{2}{*}{ MOS } & Male & 6.7 & 8.0 & 15.4 & 18.7 & 21.1 & 14.0 \\
\hline & Female & 5.1 & 9.2 & 8.9 & 17.9 & 22.4 & 19.0 \\
\hline \multirow[t]{2}{*}{ WAR } & Male & 3.9 & 2.6 & 13.3 & 2.7 & 23.0 & 5.7 \\
\hline & Female & 5.5 & 5.5 & 7.5 & 6.0 & 24.5 & 6.1 \\
\hline \multirow[t]{2}{*}{ BER } & Male & 6.8 & 10.2 & 10.8 & 11.8 & 21.2 & 13.1 \\
\hline & Female & 9.0 & 19.1 & 6.5 & 29.0 & 12.1 & 18.7 \\
\hline \multirow[t]{2}{*}{ ZAG } & Male & 4.3 & 19.6 & 5.9 & 25.0 & 9.3 & 33.3 \\
\hline & Female & 6.0 & 34.9 & 13.8 & 50.0 & 32.3 & 38.7 \\
\hline \multirow[t]{2}{*}{ DEL } & Male & 9.0 & 12.6 & 10.7 & 13.6 & 23.0 & 23.0 \\
\hline & Female & 4.2 & 20.8 & 5.7 & 14.9 & 13.6 & 28.8 \\
\hline \multirow[t]{2}{*}{ HON } & Male & 3.1 & 9.2 & - & 12.5 & 2.4 & 21.4 \\
\hline & Female & 1.0 & 14.0 & 2.4 & 15.9 & 5.3 & 21.1 \\
\hline \multirow[t]{2}{*}{ TOK } & Male & 15.6 & 20.0 & 16.9 & 19.1 & 34.0 & 22.6 \\
\hline & Female & 2.3 & 16.1 & 20.3 & 16.2 & 17.9 & 17.9 \\
\hline \multirow[t]{2}{*}{ HAV } & Male & 3.4 & 6.8 & 4.3 & 6.5 & 15.6 & 7.8 \\
\hline & Female & 1.2 & 8.1 & 8.8 & 11.0 & 10.0 & 15.0 \\
\hline \multirow[t]{2}{*}{ OKL } & Male & 8.6 & 13.7 & 11.8 & 16.2 & 37.0 & 11.1 \\
\hline & Female & 8.4 & 15.2 & 11.9 & 17.9 & 14.3 & 16.3 \\
\hline \multirow[t]{2}{*}{ ARI } & Male & 6.3 & - & 9.8 & 9.8 & 23.1 & 23.1 \\
\hline & Female & 6.3 & 4.2 & 6.0 & 7.5 & 18.2 & 6.1 \\
\hline \multirow[t]{2}{*}{ BUL } & Male & 2.1 & 8.5 & 2.9 & 16.2 & 16.1 & 21.4 \\
\hline & Female & - & 17.9 & 2.3 & 14.9 & 9.0 & 19.4 \\
\hline
\end{tabular}

Table 31. Small-vessel disease univariate associations; pooled data

\begin{tabular}{|c|c|c|c|c|}
\hline & \multicolumn{2}{|l|}{ SVDE } & \multicolumn{2}{|l|}{ SVDK } \\
\hline & \multicolumn{2}{|l|}{ Corr. coeff } & \multicolumn{2}{|l|}{ Corr. coeff } \\
\hline \multicolumn{5}{|l|}{ Men } \\
\hline Age & - & - & 0.030 & $X$ \\
\hline Duration & 0.445 & XXX & 0.174 & $\mathrm{XXX}$ \\
\hline Systolic BP & 0.158 & $\mathrm{XXX}$ & 0.227 & XXX \\
\hline Diastolic BP & 0.034 & $\mathrm{X}$ & 0.163 & $\mathrm{XXX}$ \\
\hline Hypertension & 0.079 & $\mathrm{XXX}$ & 0.190 & $\mathrm{XXX}$ \\
\hline $\mathrm{BMI}$ & -0.170 & $\mathrm{XXX}$ & - & - \\
\hline Smoking & - & - & 0.036 & $\mathrm{X}$ \\
\hline Treatment & 0.354 & XXX & 0.093 & $\mathrm{XXX}$ \\
\hline Cholesterol & - & - & 0.079 & XXX \\
\hline \multicolumn{5}{|l|}{ Women } \\
\hline Age & 0.070 & $\mathrm{XXX}$ & - & - \\
\hline Duration & 0.422 & $\mathrm{XXX}$ & 0.121 & $\mathrm{XXX}$ \\
\hline Systolic BP & 0.137 & $\mathrm{XXX}$ & 0.183 & XXX \\
\hline Diastolic BP & - & - & 0.140 & $\mathrm{XXX}$ \\
\hline Hypertension & 0.108 & $\mathrm{XXX}$ & 0.152 & $\mathrm{XXX}$ \\
\hline BMI & -0.136 & $\mathrm{XXX}$ & - & - \\
\hline Smoking & - & - & - & - \\
\hline Treatment & 0.327 & $\mathrm{XXX}$ & 0.086 & $\mathrm{XXX}$ \\
\hline Cholesterol & 0.054 & $\mathrm{XX}$ & 0.155 & $\mathrm{XXX}$ \\
\hline
\end{tabular}

Only statistically significant correlations are shown. Key: $\mathrm{X}=0.01$ $<p<0.05 ; \mathrm{XX}=0.001<p<0.01 ; \mathrm{XXX}=p<0.001$
Table 32. Standardised $\beta$-coefficients for regression of analytical variables on small-vessel disease endpoints. Pooled data

\begin{tabular}{|c|c|c|c|c|}
\hline \multirow{2}{*}{ Independent variables } & \multicolumn{2}{|c|}{ SVD Eye } & \multicolumn{2}{|c|}{ SVD Kidney } \\
\hline & Men & Women & Men & Women \\
\hline Age (years) & -0.03 & $0.04^{a}$ & -0.02 & -0.03 \\
\hline Duration (years) & $0.33^{b}$ & $0.33^{\mathrm{b}}$ & $0.13^{\mathrm{b}}$ & $0.09^{\mathrm{b}}$ \\
\hline $\begin{array}{l}\text { Systolic blood } \\
\text { pressure }(\mathrm{mmHg})\end{array}$ & $0.15^{b}$ & $0.12^{b}$ & $0.22^{\mathrm{b}}$ & $0.17^{\mathrm{b}}$ \\
\hline $\begin{array}{l}\text { Body mass index } \\
\left(\mathrm{kg} / \mathrm{m}^{2}\right)\end{array}$ & $-0.08^{b}$ & $0.04^{\mathrm{b}}$ & 0.01 & 0.03 \\
\hline Smoking & 0.02 & 0.02 & $0.05^{\mathrm{b}}$ & 0.00 \\
\hline Treatment & $0.18^{\mathrm{b}}$ & $0.16^{\mathrm{b}}$ & 0.03 & $0.04^{\mathrm{a}}$ \\
\hline Cholesterol & 0.01 & 0.01 & $0.04^{\mathrm{a}}$ & $0.10^{b}$ \\
\hline Multiple $\mathrm{R}^{2}$ & 0.24 & 0.22 & 0.08 & 0.06 \\
\hline
\end{tabular}

The WHO Multinational Study tried to resolve some of these problems by formulating strict definitions of diabetic populations and their sources. The sampling frame minimized visit-frequency bias by collecting balanced samples stratified within the selected age-band (35-54 years), according to sex and known duration of diabetes. Even so, where sampling was based upon clinic lists, non-attenders were unrepresented. The crosssectional design, disciplined use of standardized questionnaires, agreement upon systematic methods for recording physical characteristics and clinical observations, and coordination of certain examination data and laboratory methods through WHO Geneva, provide a scope and degree of comparability of investigation of vascular disease in diverse populations of diabetic which was not previously available. Nevertheless, despite the special measures taken, the WHO Multinational Study has a number of shortcomings. These deficiencies will be more fully considered in the appropriate sections of the discussion. Furthermore, we only report prevalence data, with its inherent restrictions on the interpretation and comparison of rates and relationships. Incident events of vascular disease will be collected in a second phase of the study: this follow-up will meet some of the objections and reservations referred to above.

\section{Recruitment}

Some centres had difficulty recruiting to particular stratifying subgroups, especially those for younger patients of longer duration. The total numbers of participants approximated the goal of 250 men and 250 women per centre, except for a shortfall in Arizona, where virtually all available eligible persons were recruited, and the overshoot in Oklahoma.

There was no evidence that comparisons between groups were distorted by selective under- or over-representation of patients with endpoint vascular variables (as assessed from ordinary clinical records) among the noncompliant. 
Table 33. Multiple regression analysis: values of $\beta$ coefficients and $F$ statistics ${ }^{a}$. Dependent variable $=$ SVDE

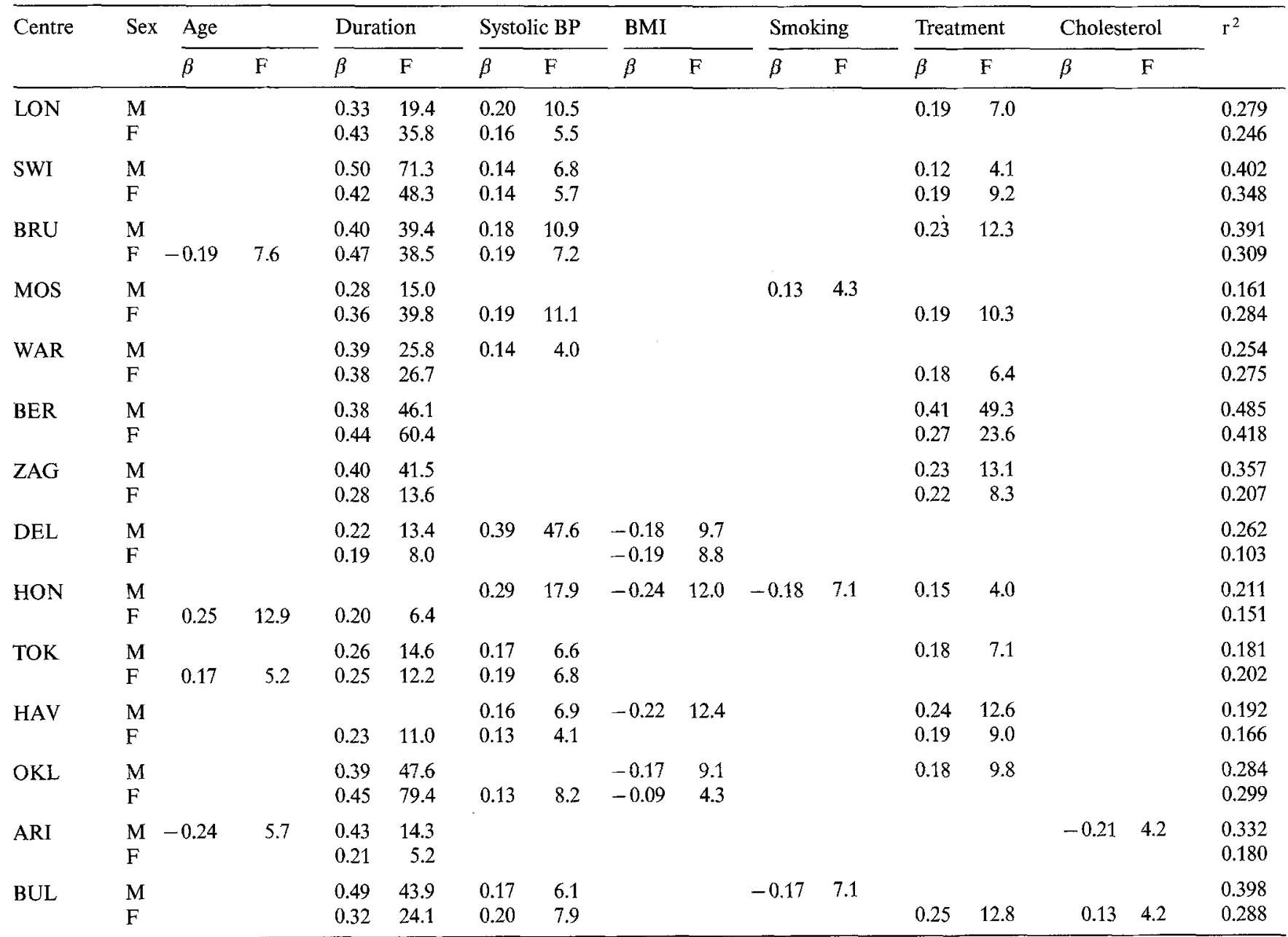

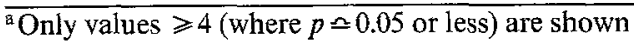

The salient features of the national samples, noted in the results, show some proportionate over-representation of the older patient categories, and a similar weighting towards diabetics of short duration at the expense of those of long duration. In some centres (e.g. Oklahoma and Japan) this deficiency probably reflects a relative scarcity of this 'type' of diabetes by comparison with European populations.

To an extent, disparities in age composition can be allowed for statistically, but this approach is less satisfactory for differences in diabetes duration. Certain endpoint components (e.g. retinovascular proliferative change, heavy proteinuria) make their appearance relatively suddenly after 15-20 years of diabetes. They do not increase slowly and smoothly with time, either in their expressed intensity within individuals, or in the proportion of diabetic patients involved. Extrapolation to long duration is therefore difficult or impossible.

\section{Vascular 'risk factors'}

There was considerable heterogeneity among centres for many of the biometric and physical variables relat- ed to vascular disease development. However, taking all 6695 subjects, the median systolic blood pressure for men was over $135 \mathrm{mmHg}$ and for women over $140 \mathrm{mmHg}$. Thirty-four per cent of the subjects qualified for the WHO designation of hypertension (BP systolic over 160, or BP diastolic over 95, or treatment for hypertension). Slightly more than half of these (1213 or 2272 ) were receiving anti-hypertensive treatment; in only half of the treated group (546 of 1213) were pressures found to be below the hypertensive range.

In populations of non-diabetic subjects of similar age and sex distribution, Keys in the Seven Countries Study found mean systolic pressures varied between 138 and $143 \mathrm{mmHg}$ [23]; the range of $135-140 \mathrm{mmHg}$ among diabetic population samples of this study is very similar. This absence of systematically different systolic (and diastolic) pressures in diabetic compared with non-diabetic subjects was also noted in the Whitehall Study when pressures in diabetic patients (men 40-64 years, mean systolic $137 \mathrm{mmHg}$ ) were measured in ignorance of their diagnosis and at the same time as a group of approximately 18000 non-diabetic individuals (mean systolic $136 \mathrm{mmHg}$ ) [24]. There is inconsistency 
Table 34. Multiple regression analysis: values of $\beta$-coefficients and F statistics ${ }^{\mathrm{a}}$. Dependent variable - SVDK

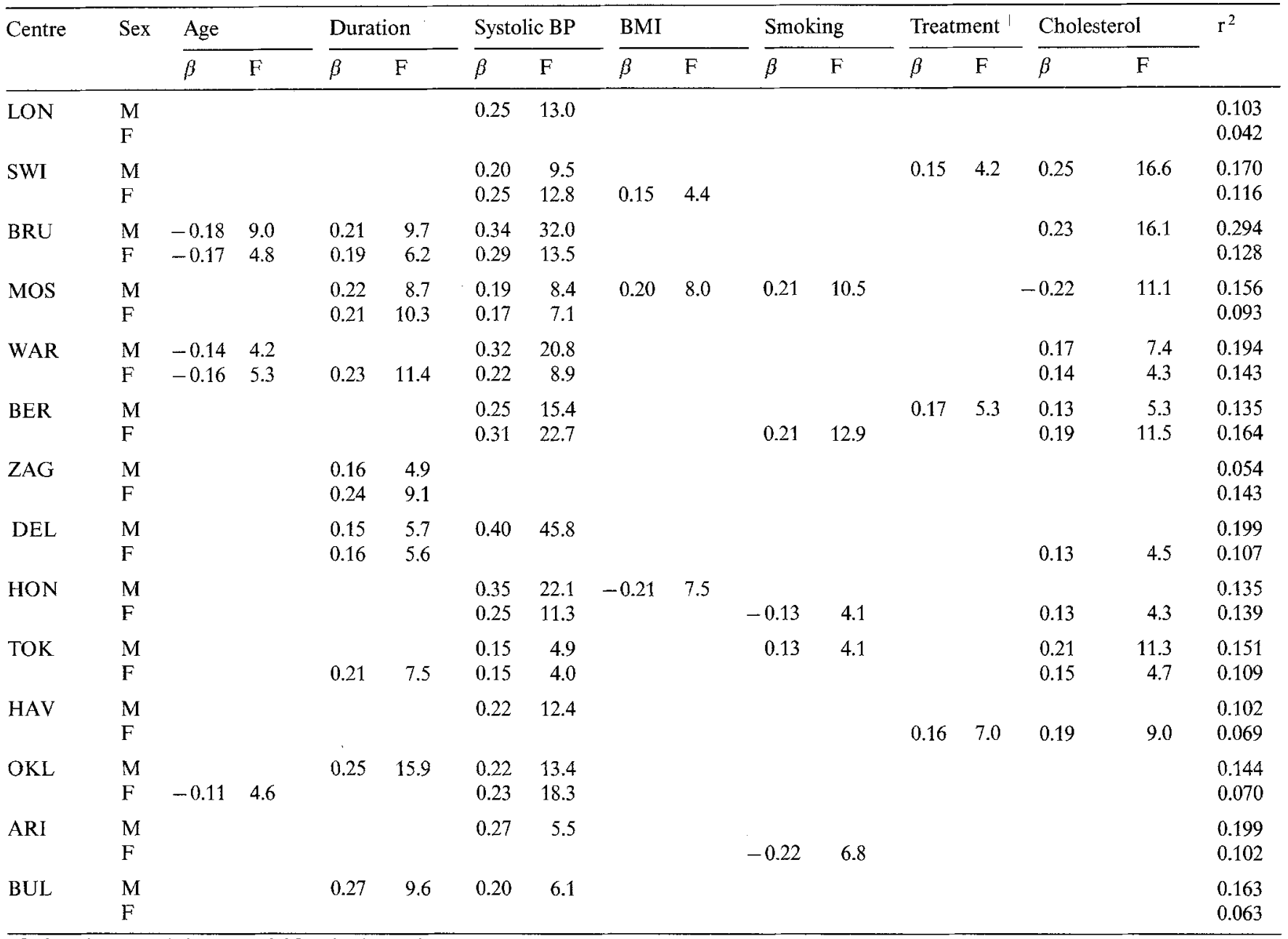

Only values $\geqslant 4$ (where $p \bumpeq 0.05$ or less) are shown

in the literature over the association of diabetes mellitus and hypertension, or blood pressure levels $[1,15]$ and it may be that there are real inter-population differences. There is also potential confounding by the association of obesity and diabetes. There is agreement, however, that elevated arterial pressure is at least as damaging in diabetics as in non-diabetics [1].

The Tokyo sample had the lowest levels of arterial pressure and was among the lowest in proportion hypertensive (WHO definition). The Brussels sample had a remarkably lower proportion of its hypertensives on blood pressure lowering therapy (17 of 145), and "normal' pressures were recorded in only four of these. Mean arterial pressures rose with age in all populations, more steeply in women than men. In all but two populations the systolic arterial pressures in the oldest women exceeded those for men.

There was much greater variation between centres in the degree of adiposity as measured by BMI. The highest value for Tokyo males fell below the mean for Oklahoma females. Mean values for BMI ranged between 22 and 26 for non-diabetics in the Seven Coun- tries Study [23], compared with the range of 22 to 33 shown here, the highest values arising from the two Amerindian populations and Moscow and Warsaw women. Conclusions and comparisons should be drawn with some caution, as BMI values may be influenced by ethnic differences. In particular, contribution of upper and lower body segments to total height and pattern of body fat distribution may differ between Oriental and Occidental individuals.

Cigarette smoking habits also show major variations between centres. Among diabetic subjects studied, a much higher proportion of men than women smoke, particularly in the heavier smoking grades. Tokyo men are the heaviest cigarette consumers, and Hong Kong, Havana, Warsaw, Zagreb, Brussels and London men approach their smoking rates. There are few comparative data concerning smoking habits of diabetic subjects and non-diabetic subjects [15]. The striking disparity between men and women in some of the populations in the present study together with similar rates of ECG abnormalities (coronary probable) is evidence against a significant role of cigarette smoking in the excess preva- 
lence of large vessel disease in diabetic patients, though here again prospective incidence data will be more informative.

Restricting cholesterol comparisons to those measured centrally, there remains a considerable variation. Mean values are relatively low from the Tokyo and Arizona samples and high from Switzerland, Zagreb, Delhi, Berlin and Havana. Mean cholesterol values rise substantially with age only among women, and there is no age-gradient in men. Comparisons with non-diabetic population values can only be tentative, since different analytical methods have been used. The overall means for the diabetic sample considerable exceeded the mean values for non-diabetic American men $5.32 \mathrm{mmol} / 1$, or $206 \mathrm{mg} / \mathrm{dl})$ and women $(5.17 \mathrm{mmol} / 1$, or $200 \mathrm{mg} / \mathrm{dl}) \mathrm{re}-$ ported by the US Lipid Research Clinic Prevalence Study for the 35-55 age range [25]. Ranking the mean cholesterol values for a centre and comparing with vascular disease prevalence did not reveal any obvious correlation.

\section{Treatment of diabetes mellitus}

Patterns of use of insulin injection, oral anti-diabetic agents and diet alone show considerable variation among the 14 centres. These differences partly reflect the variability in relative prevalence of insulin-dependent and non-insulin-dependent types of diabetic patient for the various samples. Local therapeutic philosophies in the use of insulin to achieve glycaemic control, and economic determinants of the availability for use of the various medications, may contribute to these different patterns. Even in the longest diabetes-duration group, where the great majority of true insulin-dependent diabetic patients might be expected among Europids, fewer than one half of the Delhi sample and little more than half of the Oklahoma sample were receiving insulin. Insulin use in the samples as a whole varies from as little as $20 \%$ to as much as $60 \%$. Similar variation is seen with use of oral agents, where rates range from a low of $25 \%$ to a high of $69 \%$. Only a minority of diabetics (about 16\%) used neither medication, in Hong Kong under $2 \%$. The association of mode of therapy with vascular disease is difficult to interpret, as the appearance of complications may signal intensification of therapy.

\section{Large-vessel disease prevalence}

One of the major purposes of this study was to examine the assertion that there were large differences in the degree to which atheroclerotic disease affected diabetic populations of dissimilar geographical origin. The arterial disease endpoints we have selected to evaluate can be considered individually or grouped in various ways. Data on which the estimates are based can be divided into objective evidence (e.g. centrally-analysed ECG results, limb amputation) and subjective evidence (e.g. symptom enquiry for angina, claudication, stroke, etc.). Manifestations of arterial disease can be grouped by organ system (e.g. heart, leg, brain) or can be divided into broader categories of likelihood of underlying vascular disease. We have undertaken analysis using all of these approaches. The results of all are in broad agreement, and support the hypothesis that major variations in arterial disease prevalence occur.

In the broad category of 'Total LVD', age-standardized prevalence in men was $28 \%$ and in women $36.3 \%$ for the group as a whole. The female excess stems from the high rate of 'ECG Coronary Possible' (ST, T wave abnormalities), and the higher symptom complaint rate for 'Angina Pectoris' than in diabetic men. If comment is restricted to the most objective evidence of arterial disease, 'ECG Coronary Probable' (Q wave items) occurs with approximately equal frequency in the two sexes (4.5\% and $4.2 \%$ in men and women respectively), with the composite category of 'CVD Severe' including $6.3 \%$ and $5.4 \%$ men and women respectively. On a centre-by-centre basis, overall rates of 'ECG Coronary Probable' abnormalities ranged from $0.4 \%$ in Hong Kong women to $10.5 \%$ in women from East Berlin. The Delhi sample differed sharply from the other Asian groups with rates resembling the European centres. The Oklahoma Indian sample differed sharply from the Asian groups and from the Pima Indians.

'ECG Coronary Probable' rates for women equal, or in some cases even surpass, those for men. Notable exceptions were New Delhi, Arizona and Warsaw where rates in men were 2-4 times higher. Previous reports from Warsaw on mortality rates also showed that they were lower in female diabetics who had an excess mortality rate similar to that in men [26]. One prospective study has drawn attention to the high frequency of congestive heart failure without evidence of antecedent coronary heart disease among diabetic patients, particularly in insulin-treated women, in this age group [27]. Heart failure in such diabetic patients has been attributed to diabetic cardiomyopathy [28]. Our study did not enquire about heart failure and may therefore underestimate the prevalence of severe heart disease in diabetics.

Lesser ECG abnormalities, mainly ST segment and $T$ wave items, occur frequently and greatly inflate estimates of 'Heart VD', especially when questionnairepositive 'Angina Pectoris' is also included in the category. These repolarisation abnormalities are non-specific. While they may represent ischaemic heart disease, an unknown and probably variable proportion may have other explanations, such as old myocarditis, metabolic and nutritional causes. The predictive value of these ECG abnormalities should emerge from the current prospective phase of the study.

Mean arterial pressure and plasma cholesterol were higher in all categories of LVD than in those without it. 
The relationship with adiposity and LVD endpoints was much less striking. Almost all LVD endpoints were associated with longer diabetes duration for men, but not for women, with the exception of 'Leg VD'. Current smoking habit showed strikingly little association with the prevalence of all arterial disease endpoints investigated.

In the logistic analysis, age, duration of diabetes, hypertension and BMI are independently associated with the LVD endpoints for both men and women, while smoking and cholesterol level do not show significant independent roles. The results for 'Heart VD' parallel those for 'Total LVD'. The lack of any significant association for smoking with the 'Leg VD', 'Stroke Only' and 'ECG Coronary Probable' endpoints may reflect altered habits following development of vascular disease. Prospective data will investigate this possibility further. Similar logistic analyses for triglyceride and glucose data from five of the 14 populations in this study showed that higher glyceride values in the diabetics were associated with 'Large-Vessel Disease', 'Stroke Only' and 'Leg VD' categories, while fasting glucose showed no association with 'Heart VD' [29]. Withincentre analyses showed blood pressure, followed by age, to be the most consistent independent variables associated with LVD and HVD. Significant effects of duration and BMI seen in pooled data were rarely observed within centres.

\section{Small-vessel disease prevalence}

Small-vessel disease of the eye, as defined in this study, is virtually unique to the diabetic. Renal disease (defined by proteinuria) is also included in the microvascular disease category, though it is more likely to be specific in the heavier than in light proteinuria. The frequency of almost all of the components of SVD was greater with longer duration of diabetes. Among individuals with diabetes for more than 14 years, over $5 \%$ were seriously visually disabled ('Retinopathic Blindness'). Overall, 15\% had 'Severe SVDE'. This last class corresponds essentially to proliferative retinopathy, carrying a poor prognosis for vision [30]. Only $32 \%$ of men and $38 \%$ of women with diabetes of long duration were without significant eye disease in this study.

The results for renal disease are just as striking. Proteinuria in diabetes indicates advanced renal disease, and heralds the almost inevitable development of progressive renal failure [31].

Diabetic eye and kidney disease are both associated, in a roughly proportionate way, with elevated mean systolic pressure and increased mean plasma cholesterol concentrations. The cholesterol association was more evident with kidney than with eye disease. It is not possible to distinguish between causal and consequential relationships for these data. In almost all duration-categories BMI is lowest in the most severe grades of reti- nopathy. Trivial trends occur in the reverse direction for BMI and proteinuria, an association which may in part be secondary to fluid retention accompanying renal impairment. The association of SVDE with lower BMI confirms a recent report on retinopathy in Oklahoma Indians [32]. Greater prevalence of retinopathy in the more severely hyperglycaemic, thinner or insulin-dependent diabetics may exist, and further analysis is necessary before conclusions can be drawn.

The variation in centre-by-centre enumerations for the subclasses of SVDE and SVDK could reflect true regional biological differences, but different examiners made assessments in each centre, and observer error plays an unknown role. A recent study reported a $50 \%$ error rate for the diagnosis of proliferative retinopathy by internists and diabetologists using direct ophthalmoscopy, for diagnoses established by pan-retinal photography [33]. The potential for observer variation is probably less great with proteinuria quantification, as it was more easily standardized.

When the variables age, diabetes duration, arterial pressure, BMI, smoking, diabetic treatment and cholesterol are submitted to multivariate analysis, eye disease retains its association with duration and systolic blood pressure, and the inverse association with BMI of a very low order of magnitude remains. All of these findings are independent of the mode of therapy. The multiple correlations with renal disease are all of a lower order than for the eye, and systolic blood pressure is the strongest of a very weak set of explanatory variables. Withincentre analyses resembled those using pooled data, with consistent associations of SVDE with blood pressure and duration and of SVDK with blood pressure.

Noteworthy differences in the frequencies of severe retinopathy and of heavy proteinuria were reported in the Tokyo and Hong Kong samples. This large and unexpected disparity for the two Oriental samples is unexplained. Closer analysis of the Hong Kong and Tokyo data suggests that these differences reflect dissimilarities in the prevalence of 'New Vessels', 'Large Red Lesions' and "Heavy Proteinuria' but not "Retinopathic" Blindness'.

It is difficult to make comparisons between the centres in respect of 'retinopathic blindness': in shorter-duration categories the numbers affected are very small, and the longer-duration groups differ in the proportion of 'extra-long' (more than 21 years')-duration patients included in each centre. Such a dissimilar composition in the greater-than-14-year-duration group may help to explain the wide variation $(5.7 \%$ in Warsaw men to $32.8 \%$ in Bulgarian 'men) in 'severe SVDE' prevalence. Nevertheless, comparisons within the 7-13-year-duration group show more than a tenfold variation $(0.9 \%$ to $13.5 \%$ ) in 'severe SVDE' prevalence rates. Differences in duration are unlikely to be responsible for this discrepancy in this better-defined group. Despite their imperfections, the data cast doubt upon the often-quoted impression that vulnerability to diabetic microvascu- 
lar disease is constant in diverse diabetic populations. However, to establish this clearly, more refined and standardized procedures are required. These were not generally available for the present study. Methods currently available might be justifiably applied in a more limited cross-cultural study to test the hypothesis.

\section{Implications of study}

Use of standardized methods to achieve comparability was the chief purpose of this multicentre investigation. Diabetic subjects in this study showed considerable diversity in the vascular disease type and its manifestations within individual centres, and, most provocatively, between centres. Clearly, conclusions about diabetic patients drawn from one culture cannot automatically be extrapolated to all diabetic populations.

Hypertension as defined by WHO is not only common, and often poorly controlled, in the studies of diabetic patients, but is related to the prevalence of both classes of vascular disease ascertained. Data for improved blood pressure control and the potential effect on retinopathy are lacking, but there is some evidence that the rate of progression of late renal disease can be slowed by effective control of hypertension [34].

There is an unusually strong link between adiposity and arterial disease in the group as a whole. When individual centres are examined, no relationship is seen, and in some centres the relationship is reversed. The significant association of adiposity and arterial disease may emerge artefactually, based upon the low overall frequency of arterial disease in the low-BMI subjects in Tokyo and Hong Kong, and the unexpectedly high frequency in the Oklahoma Indians. Within these and other centres, adiposity is not associated with LVD.

Failure to demonstrate an association between two factors where an effect was anticipated can easily occur in a prevalence study. Changes in habits, selective survival, and considerable heterogeneity among the participants may all contribute to this sort of error. Conversely, false conclusions may be drawn where an association is demonstrated and none was expected. The preceding paragraphs discuss each of these phenomena. Successful completion of the follow-up study, currently under way, should help to reduce these potential errors, and allow more specific comments about some of the pathophysiological mechanisms underlying vascular disease complications in diabetes mellitus.

\section{References}

1. Jarrett RJ (1984) The epidemiology of coronary heart disease and related factors in the context of diabetes mellitus and impaired glucose tolerance. In: Jarrett RJ (ed) Diabetes and heart disease. Elsevier, Amsterdam, pp 1-23

2. Robertson WB, Strong JP (1968) Atherosclerosis in persons with hypertension and diabetes mellitus. Lab Invest 18: 538-551
3. Jarrett RJ, Keen H, Grabauskas V (1979) The WHO Multinational Study of Vascular Disease in Diabetes: 1. General description. Diabetes Care 2: 175-186

4. WHO International Document (1982) Vascular disease in diabetics. Report of WHO Multinational Study of Vascular Disease in Diabetics. NCD/OND/79.4

5. Manual of Laboratory Operation, Lipid Research Clinics Program (1974) Vol 1 (DHEW Publication) [No. (NIH) 75-628]

6. Rennie IDB, Keen H, Cowhig J, Field M, Harvey M, Quartey E (1967) Evaluation of clinical methods for detecting proteinuria. Lancet 2: 489-492

7. Rose GA, Blackburn H (1968) Cardiovascular survey methods. WHO monograph Series No56, World Health Organisation, Geneva, pp 172-175

8. Blackburn H, Keys A (1960) The electrocardiogram in population studies. A classification system. Circulation 21:1160-1175

9. Walker SH, Duncan DB (1967) Estimation of the probability of an event as a function of several independent variables. Biometrica 54: 167-179

10. Keen H, Jarrett RJ (1979) The WHO Multinational Study of Vascular Disease in Diabetes: 2. Macrovascular disease prevalence. Diabetes Care 2: 187-195

11. Jarrett RJ, Keen H (1979) The WHO Multinational Study of Vascular Disease in Diabetes: 3. Microvascular disease. Diabetes Care 2: 196-201

12. Goto Y (1978) Vascular complications in diabetes in Japan. In: Miller $\mathrm{M}$ and Bennett $\mathrm{PH}$ (eds) Advances in metabolic disorders, Vol 9. Academic Press, New York, pp 167-200

13. Shaper AG, Lee KT, Scott RF, Goodale F, Thomas WA (1962) Comparison of adipose tissue from East Africa and the United States with different frequencies of myocardial infarction. Am J Cardiol 10: 390-406

14. Greenwood BM, Taylor JR (1968) The complications of diabetes in Nigerians. Trop Geog Med 20: 1-12

15. Jarrett RJ, Keen H, Chakrabarti R (1982) Diabetes, Hyperglycaemia and Arterial Disease. In: Keen H, Jarrett J (eds) Complications of diabetes. Edward Arnold, London, pp 179-203

16. West KM (1978) Atherosclerosis and related disorders. In: West KM (ed) Epidemiology of diabetes and its vascular lesions. Elsevier, New York Oxford, pp 353-402

17. Epstein FH, Ostrander LD, Johnson BC, Payne MW, Hayner NS, Keller JB, Francis T, Jr (1965) Epidemiological studies of cardiovascular disease in a total community - Tecumseh, Michigan. Ann Intern Med 62: 1170-1187

18. Kannel WB, McGee DL (1979) Diabetes and glucose tolerance as risk factors for cardiovascular disease: The Framingham Study. Diabetes Care 2: 920-926

19. Reid DD, Brett GZ, Hamilton PJS, Jarrett RJ, Keen H, Rose G (1974) Cardiorespiratory disease and diabetes among middleaged male civil servants. Lancet 1:469-473

20. Medalie $\mathrm{JH}$, Goldbourt U (1976) Angina pectoris among 10000 men: 2. Psychosocial and other risk factors as evidenced by a. multivariate analysis of a five-year incidence study. Am J Med 60: 910-921

21. Kannel WB, McGee DL (1979) Diabetes and cardiovascular disease. The Framingham Study. J Am Med Ass 241: 2035-2038

22. Rose G, Keen H, Jarrett J (1979) Epidemiologic methods in diabetic macrovascular disease. Diabetes Care 2: 91-97

23. Keys A (1980) Seven countries: a multivariate analysis of death and coronary heart disease. Harvard University Press Harvard, Cambridge, London, $\mathrm{p} 44$

24. Jarrett RJ, Keen H, McCartney M, Fuller JH, Hamilton PJS, Reid DD, Rose G (1978) Glucose tolerance and blood pressure in two population samples: their relation to diabetes mellitus and hypertension. Int J Epidemiol 7: 15-24

25. The Lipid Research Clinics Population Studies Data Book (1980) Vol I. The Prevalence Study (DHHS publication No (NIH) 80-1527)

26. Królewski AS, Czyzyk A, J̀aneczko D, Kopczyński J (1977) Mortality from cardiovascular diseases among diabetics. Diabetologia $13: 345-350$ 
27. Kannel WB, Hjortland M, Castelli WP (1974) Role of diabetes in congestive heart failure: The Framingham Study. Am J Cardiol 34: $29-34$

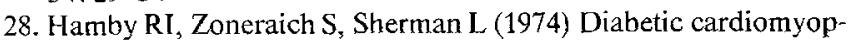
athy. JAMA 229: 1749-1754

29. West KM, Ahuja MMS, Bennett PH, De Acosta OM, Fuller JH, Grab B, Grabauskas V, Jarrett J, Kinori K, Keen H, Królewski AS, Miki E, Schliack V, Teuscher A (1982) Relationship of macroangiopathy to blood glucose, triglyceride, and other risk factors in diabetics of nine populations. Diabetologia 22: 412-420

30. Diabetic Retinopathy Study Research Group (1979) Four risk factors for severe visual loss in diabetic retinopathy. Arch Ophthalmol 97: 654-655

31. Knowles HC (1974) Magnitude of the renal failure problem in diabetics. Kidney Int (Suppl 1) 6:2-7

32. West KM, Erdreich LJ, Stober JA (1980) A detailed study of risk factors for retinopathy and nephropathy in diabetes. Diabetes 29 : 501-508

33. Sussman EJ, Tsiaras WG, Soper KA (1982) Diagnosis of diabetic eye disease. JAMA 247: 3231-3237

34. Parving HH, Andersen AR, Smidt UM, Früisberg B, Svendsen PA (1981) Reduced albuminuria during early and aggressive antihypertensive treatment of insulin-dependent diabetic patients with diabetic nephropathy, Diabetes Care 4: 459-463

Address for correspondence:

Prof. H. Keen

Unit for Metabolic Medicine

4th floor, Hunt's House

Guy's Hospital

London SE1 9RT

UK

Detailed protocols may be obtained from:

M. O. in charge

NCD/ONCD

World Health Organisation

Via Appia

Geneva 27

Switzerland

\section{Appendix 1.}

$\begin{array}{ll}\text { Members of the Drafting Group } \\ \text { B. Grab } & \text { WHO, Geneva } \\ \text { V. Grabauskas } & \text { WHO, Geneva } \\ \text { R.J.Jarrett } & \text { London, UK } \\ \text { H. Keen } & \text { London, UK } \\ \text { A. Teuscher } & \text { Bern, Switzerland } \\ \text { P.W.F. Wilson } & \text { Bethesda, MD, } \\ & \text { USA }\end{array}$

Members of Coordinating Group

B. Grab WHO, Geneva

V.Grabauskas WHO, Geneva

R.J.Jarrett London, UK

H. Keen London, UK

V. Khatchatourov WHO, Geneva

K.M.West Oklahoma, USA

List of centres and investigators

1. London, United Kingdom (LON)

Investigators: JH Fuller, RJ Jarrett, H Keen, RB Smith, PJ Watkins

2. Bern, Switzerland (SWI)

Investigator: A Teuscher
3. Brussels, Belgium (BRU)

Investigator: J Pirart

4. Moscow, USSR (MOS)

Investigator: A Mazovetsky

5. Warsaw, Poland (WAR)

Investigators: A Czyzyk, A Krolewski

6. Berlin, GDR (BER)

Investigators: E Raskovic, V Schliack

7. Zagreb, Yugoslavia (ZAG)

Investigators: I Aganovic, A Skrabalo, A Stavljenic

8. New Delhi, India (DEL)

Investigator: MMS Ahuja

9. Hong Kong (HON)

Investigator: RTT Yeung

10. Tokyo, Japan (TOK)

Investigators: K Kosaka, E Miki

11. Havana, Cuba (HAV)

Investigators: O Mateo de Acosta, S Amaro, O Diaz, M Hang,

X Quesda

12. Oklahoma, USA (OKL)

Investigator: KM West

13. Phoenix, Arizona, USA (ARI)

Investigators: PH Bennett, PJ Savage

14. Sofia, Bulgaria (BUL)

Investigator: D Andreev

Appendix 2. Non-core information collected, by Centre

Centre

London, UK 1. Plasma lipids and lipoprotein cholesterol fractions.

2. Haemostatic factors: comparison with two matched non-diabetic populations.

3. Dietary study in diabetic sample compared with matched control sample.

Bern, Switzer- 1. Blood examination: fasting glucose and triglyceride land values.

2. Urine testing for protein, glucose, acetone, nitrogen. Protein determination by enzymatic versus sulphosalicylic methods.

3. Alcohol intake

4. Clinical ECG evaluation against Minnesota Coding findings.

5. Fundus photographs in a small sample.

Brussels, 1. Neuropathy, vascular disease determinations.

Belgium 2. Insulin dependence.

3. Blood sugar control.

4. Weight.

(each of the above was determined as part of a larger surveillance project for disease in diabetics in this population).

Moscow, 1. Serum triglycerides.

USSR 2. Family history of diabetes.

3. Skinfold thickness.

4. Social-psychological-behavioural characteristics: comparison with matched non-diabetic sample.

5. Non-responders study.

6. Prospective mortality study.

7. Fasting blood glucose.

8. Special study on urine protein.

9. Retinal photography.

10. Studies on non-diabetic control. 
Berlin, GDR 1. Dietary survey.

2. Peripheral vascular disease study.

3. Neuropathy study on selected patients.

4. Fatty acid spectra of plasma cholesterol esters and plasma triglyceride.

5. Control studies in 300 non-diabetics.

Zagreb, 1. Fasting plasma triglycerides.

Yugoslavia 2. PAS lymphocyte index

3. Oscillographic leg examinations

New Delhi, 1. Dietary survey.

India

2. Study on vascular disease in relation to control of diabetic state (hyperglycaemia).

\section{Hong Kong NONE}

Tokyo, Japan 1. Fasting blood sugar

Havana, Cuba 1. Dietary survey.

2. Plasma triglycerides and lipoproteins.
3. Identical cardiovascular ascertainment of a matched non-diabetic control population.

Oklahoma, 1. Fasting blood glucose and triglycerides and

USA detailed studies on lipoproteins and apolipoproteins in diabetics and controls.

2. Skinfold thickness and previous weight over lifetime.

3. Family history of diabetes in diabetics and controls.

4. Detailed study of obesity in diabetics and controls.

5. X-ray examination of chest.

6. Cardiovascular survey.

7. Dietary survey.

Phoenix, USA 1. Retinal photography

2. Serum lipids

3. Glucose tolerance tests in diabetics and controls

4. Skinfold thickness

5. X-ray examination of chest and leg

Sofia,

Bulgaria
1. Plasma triglycerides and lipoproteins 